Abstracts from the

\title{
Abstracts from the NIH Office of Research on Women's Health 2018 Annual BIRCWH Meeting - Building Interdisciplinary Research \\ Careers in Women's Health November 28, 2018
}

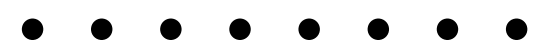




\title{
2018 Annual Meeting of the Building Interdisciplinary Research Careers in Women's Health Program
}

\author{
Lisa Begg, DrPH, RN and Melissa Ghim, PhD
}

The Office of Research on Women's Health (ORWH), National Institutes of Health (NIH), is hosting the annual meeting for its signature career development program on November 28, 2018. The program, Building Interdisciplinary Research Careers in Women's Health (BIRCWH), provides NIH funding to academic institutions that have developed scientifically competitive, mentored career development programs for junior faculty to develop their independent research careers.

ORWH developed and implemented the BIRCWH institutional career development program in 1999 to increase the number of women's health researchers working in an interdisciplinary environment. The BIRCWH Program is built around three pillars: strong mentoring, interdisciplinary research, and career development. The scholars supported by these awards are known as BIRCWH Scholars and have recently completed clinical training or postdoctoral fellowships. Paired with experienced, senior-level investigators comprising interdisciplinary mentoring teams, the scholars conduct basic, translational, clinical, or health services research related to women's health and, where appropriate, the use of both sexes to better understand the influence of sex as a variable on health and disease.

This 2018 Annual BIRCWH Meeting will open with the Ruth L. Kirschstein Memorial Keynote address that honors the life and achievements of Dr. Ruth L. Kirschstein, who was an exceptional scientist, mentor, and health administrator. In 1974, she became the first woman to direct an NIH institute, the National Institute of General Medical Sciences, a post she held until 1993. She then served as Principal NIH Deputy Director, and twice served as NIH acting director. In recognition of her leadership and support of NIH-funded training programs, the National Research Service Award (NRSA) Program was renamed to honor Dr. Kirschstein's career, service to the nation, and commitment to future generations of scientists. Dr. Kirschstein was also a member of the Institute of Medicine, and a Fellow of the American Academy of Arts and Sciences.

The Keynote address will be followed by a panel discussion on integrating Sex as a Biological Variable (SABV) into the education and training curricula of Scholars at BIRCWH institutions, to ensure its full consideration in research design, analysis, and reporting. The panel will be composed of BIRCWH Principal Investigators and other subject matter experts. Information regarding currently available curricula will be discussed to develop shared resources on SABV and sex/gender differences for all investigators, and to connect to interested collaborators.

To showcase the research achievements of the BIRCWH Programs, four BIRCWH Scholars were selected to present their research at the podium following the panel session. Their abstracts are listed first in the compendium below and represent the highly significant work of the BIRCWH Scholars overall. The topics of the oral presentations range from basic to clinical, from female vascular aging, to development of chronic pain in major thermal burn injury, to regulation of inflammation, to sex differences underlying schizophrenia. This exciting diversity of research projects are in areas crucial to the advancement of the health of women and reflect the high caliber of investigators being trained in these BIRCWH Programs.

The remaining BIRCWH Scholar abstracts will be presented during an afternoon Poster session. These posters represent many scientific areas that broadly fall within women's health and sex differences research, and include public health topics, basic, clinical, and translational science projects. These are highly meritorious projects, and their presentation should provide an important scientific networking opportunity. Additional guidance on grantsmanship issues and ways that Scholars can advance their research careers are provided in a mentoring session, attended by several NIH Institute and Center program directors and scientists. It is with great pleasure that we introduce their projects below in advance of the 2018 Annual BIRCWH Meeting.

The full list of the BIRCWH programs and additional information about the 2018 Annual BIRCWH Meeting can be found at: https:// orwh.od.nih.gov/career-development/building-interdisciplinaryresearch-careers-womens-health-bircwh

Both Dr. Begg and Dr. Ghim work as scientific administrators in the NIH Office of Research on Women's Health. For further information on the BIRCWH Program, please contact Dr. Begg at:beggl@od.nih.gov,orDr.Ghimat:ghimm@nih.gov. 


\section{BIRCWH Presentation Abstracts}

\section{O-1: Loss of Vascular Angiotensin II Type 2 Receptor Contributes Specifically to Female Vascular Aging}

Jennifer J. DuPont (presenting author), Rachel M. Kenney, Seung K. Kim, Mark J. Aronovitz, and Iris Z. Jaffe

Molecular Cardiology Research Institute, Tufts Medical Center, Boston, MA

Background and Objectives: Cardiovascular disease (CVD) is the leading cause of death in women. Aged women have greater vascular stiffness and vasomotor dysfunction vs. aged men. However, the mechanisms mediating these differences are unclear. The hormone angiotensin II promotes vascular aging via the AngII type 1 receptor (AT1R). However, AngII also acts through the AngII type 2 receptor (AT2R), which is vasoprotective. Previous studies support sex and age-related differences in AT2R function in the kidney. However, the role for vascular AT2R in sex differences in aging is unknown. Therefore, we aimed to determine the role of AT2R in sex differences in vascular aging.

Methods and Results: We measured aortic gene expression and vascular stiffness, AT1R-mediated vasoconstriction and AT2R-mediated vasodilation in mesenteric resistance vessels from 3, 12 ("middle- aged"), and 18 month ("elderly") male and female C57/B16 mice. Males develop increases in vascular stiffness at 12 months vs. 18 months in females. Further, our results show that inhibition of AT2R increases vascular stiffness in 12 month old females. AT1R-mediated vasoconstriction increases at 12 months in males, vs. 18 months in females. AT2R expression and function is enhanced in 3 month females and is reduced with age in females, not males.

Conclusions: Our data indicate sex differences in the time course and mechanisms of vascular aging. Specifically, these data suggest that reductions in AT2R expression and function contribute to female vascular aging and that AT2R activation may represent a novel therapeutic strategy in the treatment of CVD in the aging female population.

\section{O-2: Regulation of Inflammatory Cytokines by Pre-Exposure to Elevated Temperatures}

Simmie L. Foster (presenting author), ${ }^{1,2,3}$ Abby Dutton, ${ }^{3}$ Corey Seehus, ${ }^{3}$ and Clifford J. Woolf ${ }^{3,4}$

${ }^{1}$ Department of Psychiatry, Harvard Medical School, Boston, MA; ${ }^{2}$ Depression Clinical Research Program, Massachusetts General Hospital, Boston, MA; ${ }^{3}$ FM Kirby Neurobiology Center, Boston Children's Hospital, Boston, MA; and ${ }^{4}$ Department of Neurobiology, Harvard Medical School, Boston, $M A$

Background: Inflammation has a major role in many diseases that are more prevalent in women, including autoimmunity, chronic pain and major depressive disorder. Anti-inflammatories have shown some efficacy in these disorders. Elevated body temperature (fever) is a hallmark of systemic inflammation; at the onset of an inflammatory response, IL- $1 \beta$, a prototypical inflammatory cytokine, acts on the hypothalamus to raise the temperature set point. Conversely, hyperthermia-elevating body temperature to febrile range- - has been proposed to ameliorate inflammation and used to treat multiple inflammatory disorders including MDD, but the mechanisms remain unclear.

Objectives: Using an in vitro model, we aimed to test the hypothesis that prior exposure to elevated temperature modifies the ability of macrophages to produce inflammatory mediators, and asked at what levels this modification may occur.

Methods: We exposed murine bone-marrow-derivedmacrophages to febrile range temperature $\left(41.5^{\circ} \mathrm{C}\right)$ for one hour or left them at $37^{\circ} \mathrm{C}$, then stimulated with various inflammatory inducers, including lipopolysaccharides and ATP. We tested the treated macrophages for production of IL- $1 \beta$ and other inflammatory and heat-induced mediators.

Results: We found that exposure to elevated temperature prior to stimulation with microbial stimuli inhibits production of the fever-inducing cytokine IL- $1 \beta$ at the level of transcription and secretion. We also found that heat exposure inhibits components of the complex necessary for processing and secretion of IL- $1 \beta$.

Conclusion: Our results suggest prior heat exposure may particularly target IL- $1 \beta$, a potent inducer of fever, providing a potential mechanism-feed-back regulation-for the antiinflammatory effect of hyperthermia treatment.

\section{0-3: Sex Differences in Chronic Pain Development Following Major Thermal Burn Injury}

Matthew C. Mauck (presenting author), Sarah Linnstaedt, Christopher Sefton, Shan Yu, and Samuel A. McLean

Institute for Trauma Recovery, Department of Anesthesiology, University of North Carolina, Chapel Hill

Background and Objective: Annually, 11 million burn injuries occur worldwide, and chronic pain is a common, co-morbid outcome. Following traumatic injury, females are disproportionately affected by chronic pain and evidence suggests low $17 \beta$ estradiol (E2) may be involved in pain pathogenesis. However, no studies have examined sex differences or the role of E2 in chronic pain development following Major Thermal Burn Injury (MThBI). To fill this gap, we 1) measured sex-specific chronic pain severity following MThBI and 2) among females, determined the relationship between E2 levels at time of MThBI and chronic pain severity.

Methods: We conducted a prospective, multicenter study of MThBI survivors, defined as those with $\leq 30 \%$ total body surface burned. Pain severity was assessed over 1 year following MThBI via a 0-10 numeric rating scale. Plasma was collected within 72 hours of MThBI and E2 levels measured in females by immunoassay. We used linear regression models to measure the relationship between sex and chronic pain severity, and repeated measures mixed models to evaluate the relationship between E2 and chronic pain severity in females.

Results: 96 participants were enrolled, 24 (25\%) females and $72(75 \%)$ males. Female sex predicts greater chronic pain 
severity at 3 months $(\beta=2.3 \mathrm{p}=.003), 6$ months $(\beta=1.7$, $\mathrm{p}=.010)$, and 1 year $(\beta=1.7, \mathrm{p}=.002)$ following MThBI. Among females, initial circulating plasma E2 concentration and chronic pain severity were inversely related following MThBI $(\beta=-.016, \mathrm{p}=.006)$.

Conclusions: Compared to males, females experience a greater burden of chronic pain following MThBI. Among females, circulating E2 may protect against chronic pain following MThBI.

\section{O-4: Neural Mechanisms Underlying Higher Rates of Psychotic and Mood Symptoms in Females with Schizophrenia}

Laura M. Tully (presenting author), ${ }^{1}$ Ana-Maria Iosif, ${ }^{2}$ Lauren N. Zakskorn, ${ }^{1}$ Amanda Guyer, ${ }^{3}$ and Cameron S. Carter ${ }^{1}$

${ }^{1}$ Department of Psychiatry and Behavioral Sciences, University of California, Davis; ${ }^{2}$ Department of Public Health Sciences, Division of Biostatistics, University of California, Davis; and ${ }^{3}$ Department of Human Ecology, Center for Mind and Brain, University of California, Davis

Background: Females with schizophrenia (SZ) report more psychotic and mood symptoms. Further, in females, psychotic symptom severity relates to mood symptom severity, indicating a link between psychosis and emotional responses not observed in SZ males. One potential mechanism is cognitive control of emotion $(\mathrm{CCoE})$ : the self-regulation of emotions and their influence on behavior, mediated by a frontal-limbic network involving the lateral and medial prefrontal cortices and amygdala. Sex differences in $\mathrm{CCOE}$ in healthy individuals have not been examined, nor have relationships to sex differences in SZ symptomatology.

Objective: To identify sex differences in the frontal-limbic network during $\mathrm{CCoE}$ and their relationship to symptoms in females with SZ.

Methods: During fMRI, 21 healthy (11 female) and 17 SZ (10 female) participants completed the Social Evaluation Task (SET), in which participants react to or down-regulate their emotional responses to negative social evaluation (NSE) and self-report their negative emotion on a 1-to-5 scale ( $1=$ not at all negative; $5=$ very negative). Participants also completed psychosocial symptom assessments.

Results: Preliminary SET results suggest that compared to healthy males, healthy females demonstrate greater reactivity to (standardized difference $=1.45$ ) and greater regulation of (standardized difference $=1.18$ ) NSE. Compared to SZ males, SZ females demonstrate greater reactivity to (standardized difference $=0.66$ ) but reduced regulation of NSE ( standardized difference $=-0.30$ ).

Conclusions: Females may be more reactive to NSE, and SZ females may have impaired regulation of NSE compared to SZ males. Future work will examine associated frontal-limbic activity, relationships with symptoms, and within-sex comparisons of SZ to healthy participants. 


\section{BIRCWH Poster Abstracts}

\section{P-1: Daily Experiences of Posttraumatic Stress and Non-Medical Prescription Opiod Use: Examining Sex Differences}

Christal L. Badour (presenting author)

Department of Psychology, University of Kentucky

Background: Adults with posttraumatic stress disorder (PTSD) are 1.5-1.8 times more likely than those without PTSD to meet criteria for non-medical prescription opioid use (NMPOU) disorder. Compared to men, women are twice as likely to meet criteria for PTSD, and are more likely to be prescribed opioids in higher doses and for longer durations. Women are also more likely to report using prescription opioids to cope with negative affect and physical pain, to endorse prescription opioids as their primary substance of abuse, and to experience greater functional impairment and psychiatric comorbidity when seeking treatment for NMPOU disorder. Therefore, we aimed to determine the role of AT2R in sex differences in vascular aging.

Objectives: This prospective cohort study aims to examine sex differences in the relationship between PTSD symptoms and NMPOU over time.

Methods: 60 adults (30 female) with clinical or subthreshold PTSD and NMPOU during the past month will complete measures of PTSD symptoms and NMPOU daily for four weeks.

Results: Data collection is ongoing. Available results from 14 women found that severity of PTSD- negative cognitions/mood symptoms was associated with increased frequency of NMPOU, while results from 7 men found that stability (but not severity) of PTSD-negative cognitions/mood and PTSD- avoidance symptoms over the four week period was associated with increased frequency of NMPOU.

Conclusions: Preliminary findings point to important sex differences in how PTSD and NMPOU are functionally related over time. These findings highlight the importance of assessing for both severity and course of PTSD symptoms in relation to NMPOU behavior. Final results of this study will inform tailored interventions for co-occurring NMPOU and PTSD.

\section{P-2: Gender Differences Across Social, Behavioral, and Mental Determinants of Cardiovascular Health Among U.S. Indigenous Peoples: A Systematic Review}

Catherine E. Burnette (presenting author), ${ }_{2}^{1}$ Kristi Ka'apu, K., ${ }^{1}$ Jessica Liddell, $^{2}$ and Jenn Miller Scarnato ${ }^{2}$

${ }^{1}$ Tulane University School of Social Work; and ${ }^{2}$ Tulane University City Community, and Culture PhD Program

Background: Cardiovascular disease (CVD) and diabetes are among the top causes of death; yet determinants of these health outcomes among Indigenous peoples of the U.S. are limited.

Objective: The purpose of this systematic review was to identify the behavioral, mental, and social determinants of cardiovascular health among U.S. Indigenous women and men within extant research.
Methods: This systematic review includes peer-reviewed quantitative and qualitative research focusing on CVD and related health factors (e.g., diabetes, obesity, high blood pressure) of U.S. Indigenous populations of all ages published between the years of 1983-2017. Inclusion criteria delimited the search to articles that: (a) were empirical; (b) were peer reviewed; (c) addressed various outcomes of CVD and physical, behavioral, social, and mental health factors; (d) which included Indigenous peoples in their sample; and (e) occurred within the U.S. A total of 51 articles met the inclusion criteria for this systematic review.

Results: Gender predicted differences across determinants, and the patterns of these differences varied by study. Salient factors included: (a) Behavioral Factors $(\mathrm{n}=20)$ : diet, obesity and cholesterol, among other physical associates (e.g., diabetes); Mental Health Factors $(\mathrm{n}=15)$ : depression, anxiety, Post-traumatic Stress Disorder (PTSD)/Trauma, substance use disorder (SUD); Social Factors $(n=17)$ : enculturation/acculturation, exposure to violence and trauma, stress and resilience, social support, and socioeconomic factors.

Conclusion: This systematic review identifies the genderspecific connections between behavioral, mental, social determinants of health, particularly those related to CVD, diabetes, and associated health conditions among U.S. Indigenous peoples. Several culturally specific factors and understudied factors related to CVD emerged.

\section{P-3: Effects of Antiretroviral Therapy on Allele-Associated Lipoprotein(a) Levels in Women with HIV}

Enkhmaa Byambaa (presenting author), ${ }^{1}$ Erdembileg Anuurad, ${ }^{1}$ Chi- Shang Li, ${ }^{2}$ Robert Kaplan, ${ }^{3}$ Jason Lazar, ${ }^{4}$ Dan Merenstein, ${ }^{5}$ Roksana Karim, ${ }^{6}$ Brad Aouizerat, ${ }^{7}$ Mardge Cohen, ${ }^{8}$ Kenneth Butler, ${ }^{9}$ Savita Pahwa, ${ }^{10}$ Igho Ofotokun, ${ }^{11}$ Adaora A. Adimora, ${ }^{12}$ Elizabeth Golub, ${ }^{13}$ and Lars Berglund ${ }^{1}$

Departments of ${ }^{1}$ Internal Medicine and ${ }^{2}$ Public Health Sciences, University of California, Davis, CA; ${ }^{3}$ Department of Epidemiology and Population Health, Albert Einstein College of Medicine, Bronx, NY; ${ }^{4}$ Department of Cardiovascular Disease, SUNY Downstate Medical Center, Brooklyn, NY; ${ }^{5}$ Department of Family Medicine, Georgetown University Medical Center, Washington, DC; ${ }^{6}$ Department Preventive Medicine, University of Southern California, Los Angeles, CA; ${ }^{7}$ Department of Oral and Maxillofacial Surgery, New York University, New York, NY; ${ }^{8}$ Stroger Hospital, Cook County Bureau of Health Services, Chicago, IL; ${ }^{9}$ Division of Geriatric Medicine/Gerontology, University of Mississippi Medical Center, Jackson, MS; ${ }^{10}$ Miami Center for AIDS Research, University of Miami, Miami, FL; ${ }^{11}$ Department of Medicine, Infectious Diseases, Emory School of Medicine, Atlanta, GA;

${ }^{12}$ Division of Infectious Diseases, University of North Carolina at Chapel Hill, Chapel Hill, NC; and ${ }^{13}$ Department of Epidemiology, Johns Hopkins Bloomberg School of Public Health, Baltimore, MD 
Background: Lipoprotein(a) $[\mathrm{Lp}(\mathrm{a})]$ is a genetically regulated causal risk factor for cardiovascular disease (CVD).

Objectives: To test the effects of antiretroviral therapy (ART) initiation on $\mathrm{Lp}(\mathrm{a})$ levels in relation to apolipoprotein(a) [apo(a)] size polymorphism in HIV.

Methods: ART effects on total $\mathrm{Lp}(\mathrm{a})$ and allele-specific apo(a) level (ASL) - the Lp(a) level associated with the larger or smaller apo(a) allele in a given individual-were assessed in 126 HIV-seropositive women in the Women's Interagency HIV Study. ART effects were tested with a mixed-effects model across pre-ART, post-ART 1st (at 6 months) and post-ART 3rd (at 18 months) visits. Single-time point data from 120 HIVseronegative women were used for comparison.

Results: Mean age of participants was 38 years; most were African-American $(\sim 70 \%)$. Pre-ART ASL associated with the larger $(4.6 \mathrm{vs} .8 .0 \mathrm{mg} / \mathrm{dL}, \mathrm{p}=0.024)$ or smaller $(13 \mathrm{vs} .19 \mathrm{mg} /$ $\mathrm{dL}, \mathrm{p}=0.041$ ) apo(a) sizes in the HIV-seropositive group were lower compared to those in the HIV-seronegative group. PreART prevalence of a high Lp(a) level ( $\geq 30 \mathrm{mg} / \mathrm{dL})$ was lower in the HIV- seropositive vs. seronegative group (30\% vs. $46 \%$, $\mathrm{p}=0.013$ ). Post-ART both the ASL and prevalence of a high Lp(a) level was not different from the HIV-seronegative group. Initiation of ART significantly increased: 1) Lp(a) level $(p<0.0001)$ and 2) ASL associated with the larger $(\mathrm{p}=0.0003)$ or smaller $(\mathrm{p}<0.0001)$ apo(a) sizes.

Conclusions: ART initiation increased Lp(a) level and both ASLs in HIV-seropositive women. Lp(a) level, regardless of its strong genetic regulation, can be modulated by HIV/ART and could contribute to the higher CVD risk seen in HIV-seropositive individuals.

\section{P-4: Infectious Risk Perceptions Among Pregnant Women with Hepatitis C Virus Infection}

Catherine A. Chappell (presenting author), ${ }^{1,2}$ Hollis J. Laird, ${ }^{2}$ and Elizabeth E. Krans ${ }^{1,2}$

${ }^{1}$ Department of Obstetrics, Gynecology and Reproductive Sciences; University of Pittsburgh; and ${ }^{2}$ Magee-Womens Research Institute

Background: One of the most serious consequences of the opioid epidemic is the risk of bloodborne pathogen infection in women of reproductive age and perinatal transmission of hepatitis $\mathrm{C}$ virus (HCV) and human immunodeficiency virus (HIV).

Objectives: This is a qualitative study of pregnant women with opioid use disorder and HCV infection to elucidate their experiences with having HCV infection as well as their HIV risk perception through in depth interview. Each interview was transcribed and organized using Atlas.ti. An inductive approach using grounded theory was used to analyze the data which involved intense review the data from which themes and patterns were identified representing participants' experiences and perspectives.

Results: Thematic saturation was obtained after 40 interviews conducted from May 2015 to January 2016. The majority of study participants were less than 30 years of age $(57.5 \%)$, Caucasian $(95.0 \%)$, unmarried $(87.5 \%)$, unemployed $(62.5 \%)$ and receiving medical assistance $(85.0 \%)$. We identified two major themes regarding HIV or HCV perceptions: 1) HIV infection was a greater health concern that HCV infection because it is associated with greater health consequences, 2) sexual activity was perceived as a more significant risk factor for HIV than intravenous drug use, and some participant did not perceive themselves to be at risk of HIV.
Conclusions: Women at high risk of bloodborne pathogen acquisition through intravenous drug use have different perceptions of risk for HIV verses HCV. Further exploration of these perceptions can inform targeted interventions to prevent transmission of both HIV and HCV.

\section{P-5: Diabetes, Plasma Fructosamine, and Risk of Breast Cancer Mortality Among Hispanic and Non-Hispanic White Women from New Mexico}

Avonne E. Connor (presenting author), ${ }^{1}$ Kala Visvanathan, ${ }^{1}$ Stephanie D. Boone, ${ }^{2}$ Kathy B. Baumgartner, ${ }^{2}$ and Richard N. Baumgartner ${ }^{2}$

${ }^{1}$ Department of Epidemiology, Johns Hopkins Bloomberg School of Public Health; and ${ }^{2}$ Department of Epidemiology and Population Health, University of Louisville

Background: Type-2 diabetes is a significant prognostic factor for breast cancer (BC)-specific mortality. Few studies have investigated the role of hyperglycemia in the relationship between diabetes and BC mortality particularly among Hispanic women, in whom diabetes is prevalent.

Objective: We examined the associations between diabetes, plasma fructosamine- a biomarker of hyperglycemia and glycemic control, and BC-specific mortality among Hispanic and nonHispanic white (NHW) women diagnosed with invasive BC from the New Mexico Health, Eating, Activity, and Lifestyle cohort.

Methods: A total of 399 BC survivors (96 Hispanic, 303 NHW) contributed baseline data and plasma samples. Hazard ratios (HR) and 95\% confidence intervals (CI) were calculated using multivariable Cox proportional hazards regression models. Models were adjusted for age at diagnosis, ethnicity, body size, education, BC stage and treatment. The percentage change in the HRs to examine mediation due to fructosamine was computed: [HRwithout fructosamine - HRwith fructosamine)/HRwithout fructosamine -1.0$)] \times 100$.

Results: After a median follow-up time of 13 years from baseline interview to death, a total of 134 deaths occurred, of which 56 deaths were attributed to BC. Diabetes was associated with increased BC- specific mortality (HR, 2.89; 95\% CI 1.276.60); however, results were not significant stratified by ethnicity. Fructosamine was positively associated with BC-specific mortality $(\mathrm{p}=0.01)$. In mediation analysis, fructosamine explained $41 \%$ of the association between diabetes and BCspecific mortality (HRwith fructosamine $=2.09$ ).

Conclusion: Our results suggest that poor glycemic control explains a large extent of the relationship between diabetes and BC-specific mortality among Hispanic and NHW women with BC.

\section{P-6: PATHways: Comparative Effectiveness Study of Peripartum Opioid Use Disorder in Rural Kentucky}

Agatha S. Critchfield

\section{Department of Obstetrics and Gynecology, University of Kentucky College of Medicine}

Background: Perinatal opioid use disorder (OUD) is a major health concern in the U.S. with significant impact on mothers, infants, and communities. The Appalachian region has been particularly impacted. The University of Kentucky's comprehensive care model for OUD, PATHways, has demonstrated 
improvements in maternal and neonatal outcomes- yet many patients in rural Appalachia are not able to access these services. A key component of PATHways is group care and peer support. The optimal delivery modality for PATHway has yet to be determined. Telemedicine has proven utility to improve patient access to care in rural settings.

Objectives: To determine the optimal method for delivery of perinatal OUD support services to mothers receiving Medication Assisted Therapy (MAT) for OUD in rural settings.

Methods: A two-arm, twelve-site randomized cluster trial in rural prenatal care clinics to determine optimal intervention delivery methods. The two arms will be: 1) Telemedicine and 2) Group Care. A rotating eight-week patient education curriculum will be provided using these two delivery modalities. Telemedicine will include biweekly consultation with Maternal Fetal Medicine, Neonatology, Addiction Medicine, Substance Abuse Counseling. Group Care includes biweekly group sessions with a locally trained Nurse Facilitator and Peer Support Specialist. Enrolled participants will be 6-36 weeks gestation with OUD and currently receiving MAT. Follow-up is through 6 months post-partum. The primary outcomes for this trial will be medication-requiring Neonatal Abstinence Syndrome (NAS). Secondary Outcomes include: maternal relapse, maternal-infant dyad status and smoking cessation. $(\mathrm{N}=1687)$.

Results: Recently awarded funding through PCORI. Planning is underway.

Conclusions: Pending.

\section{P-7: Gender Differences in Trajectories of Biomarkers and Symptoms After Implantation of a Left Ventricular Assist Device}

Quin E. Denfeld (presenting author), ${ }^{1}$ James O. Mudd, ${ }^{2}$ Shirin O. Hiatt, ${ }^{1}$ Beth A. Habecker, ${ }^{2,3}$ and Christopher S. Lee ${ }^{4}$

${ }^{1}$ Oregon Health \& Science University School of Nursing; ${ }^{2}$ Oregon Health \& Science University Knight Cardiovascular Institute; ${ }^{3}$ Oregon Health \& Science University Department of Physiology \& Pharmacology; and ${ }^{4}$ Boston College Connell William F. Connell School of Nursing

Background and Objectives: Despite well-known etiological and pathophysiological gender differences among adults with heart failure, it is unknown how biomarkers and physical symptoms differ between women and men after implantation of a left ventricular assist device (LVAD). The purpose was to examine gender differences in trajectories of a plasma biomarker and symptoms after LVAD.

Methods: This was a secondary analysis of data collected from a study of advanced HF patients from pre- to 1, 3, and 6 months postLVAD. Plasma soluble suppressor of tumorigenicity (sST2) was assayed with an ELISA kit. Physical symptoms were assessed with the Heart Failure Somatic Perception Scale (HFSPS; higher= worse). Multiphase growth modeling (first phase $=$ pre-implantation to 1 month post-LVAD; second phase $=1$ month to 6 months postLVAD) was used to compare trajectories of sST2 and HFSPS scores between women and men.

Results: The average age of the sample $(\mathrm{n}=98)$ was $53.3 \pm$ 13.8 years, most were male $(80.6 \%)$, and most had non-ischemic etiology $(65.3 \%)$. Women had significantly lower plasma sST2 at pre-implantation $(\mathrm{p}<0.05)$ compared with men, followed by significant gender differences in the trajectories of plasma sST2 in the first phase $(\mathrm{p}<0.05)$ but not the second phase. Women had significantly higher HFSPS scores at pre-implantation $(\mathrm{p}<0.05)$ compared with men, but no significant gender differences in the first or second phase.
Conclusions: There are significant gender differences in trajectories of change in biomarkers and physical symptoms that should be taken into account for patients undergoing LVAD implantation.

\section{P-8: Timing of Tetanus, Diphtheria, and Acellular Pertussis Vaccination in a Nationwide Sample of Pregnant and Postpartum Women}

Erika L. Fuchs (presenting author), ${ }^{1}$ Jordan Westra, ${ }^{2}$ Fangjian Guo, ${ }^{1}$ Alan D. T. Barrett, ${ }^{3}$ and Yong-Fang Kuo ${ }^{2}$

${ }^{1}$ Center for Interdisciplinary Research in Women's Health and Department of Obstetrics \& Gynecology, University of Texas Medical Branch at Galveston; ${ }^{2}$ Office of Biostatistics, University of Texas Medical Branch at Galveston; and ${ }^{3}$ Department of Pathology, Sealy Center for Vaccine Development, Institute for Human Infections and Immunity, and Department of Microbiology and Immunology, University of Texas Medical Branch at Galveston

Background: Tetanus, diphtheria, and acellular pertussis (Tdap) vaccination is recommended to occur between 27 weeks and 36 weeks 6 days in each pregnancy. Little is known about demographic predictors of Tdap vaccine uptake in this window.

Objectives: To examine predictors of optimally timed prenatal Tdap vaccination.

Methods: Using Clinformatics Data Mart (CDM) electronic medical records, a retrospective cohort study was conducted using eligible (outcome at $\geq 20$ weeks) pregnancies between 2013 and 2016 in women ages 18-44. Multinomial logistic regression models with robust variance estimation to account for clustering of births within woman were conducted in Stata SE Version 15.1 with $\alpha \leq 0.05$. Age, year of delivery, race, ethnicity, Census region, insurance, and 3-digit ZIP code average household income and percent college educated were included in adjusted models.

Results: Optimally timed Tdap vaccination occurred in $17.4 \%$ of 32,802 pregnancies from 30,725 women, while $71.9 \%$ had no vaccination, $3.7 \%$ had vaccination outside of the recommended window, and $7.0 \%$ had postpartum vaccination. Few demographic variables remained associated with optimally timed uptake in all models. After adjustment, those with optimally timed Tdap vaccination were more likely to have commercial insurance than those without Tdap vaccination (relative risk ratio (RRR) 1.08, 95\% confidence interval (CI) 1.07-1.10) and postpartum vaccination (RRR 1.16, 95\% CI 1.12-1.19). Compared to those with suboptimally timed vaccination, those with optimal timing were less likely to have commercial insurance (RRR 0.96, 95\% CI 0.93, 0.99).

Conclusions: Population-level interventions are necessary to increase prenatal Tdap vaccination as demographic variables inconsistently predict uptake.

\section{P-9: Relationship Between Fetal Sex and Gestational Diabetes and Gestational Hypertension Varies by Race/Ethnicity}

Ayush Giri (presenting author), ${ }^{1,2}$ Sarah H. Jones, ${ }^{3}$ Digna R. Velez Edwards, ${ }^{1,2}$ and Katherine E. Hartmann ${ }^{1}$

${ }^{1}$ Department of Obstetrics and Gynecology, Institute for Medicine and Public Health, Vanderbilt University Medical Center; ${ }^{2}$ Vanderbilt Genetics Institute, Vanderbilt University Medical Center; and ${ }^{3}$ Division of Epidemiology, Institute for Medicine and Public Health, Vanderbilt University Medical Center 
Background: Women with male fetuses have higher risk of gestational diabetes mellitus (GDM) and gestational hypertension $(\mathrm{GH})$. Sexual dimorphism in placental contributions may explain increased risk. GDM and GH vary by race/ethnicity, so we aimed to determine if the effect of fetal sex is uniform across race/ethnic categories.

Objectives: We evaluated whether race/ethnicity modifies the relationship between fetal sex and GDM or GH using 2016 natality data from the National Center for Health Statistics.

Methods: Analyses included women with hospital-based singleton births, without prior history of diabetes and hypertension ( $\mathrm{N}=3,582,214 ; \mathrm{N}-\mathrm{GDM}=215,772 ; \mathrm{N}-\mathrm{GH}=206,559)$. We used multivariable logistic regression to evaluate the association between fetal sex and GDM while adjusting for maternal age (continuous), maternal BMI (categorical), and race/ethnicity. GH models also adjusted for GDM. We used the likelihood ratio test for interactions between race/ethnicity and fetal sex.

Results: Women with male fetuses were more likely to have GDM (odds ratio [OR]: 1.03; 95\% Confidence Interval [CI]: 1.02-1.04); and GH (OR: 1.02; 95\% CI: 1.01-1.03), than women with female fetuses. These associations varied by race/ethnicity status for GH (Minimum OR: 0.97 in Blacks; Maximum OR: 1.07 in Hispanics; P-for-interaction [all-categories] $<0.0001$ ), and GDM (Minimum: 1.01 in American Indian/Alaskan; Maximum: 1.09 in mixed race).

Conclusions: Carrying a male fetus poses a small but biologically relevant increase GDM and GH risk than carrying a female fetus. Effect modification of these associations by race/ ethnicity suggests multiple biologic phenomenon including genetic ancestry and fetal contributions likely intersect to create the conditions for GDM and GH.

\section{P-10: The Human Urinary Metabolome Changes Dramatically Across Normal Gestation}

Kathryn J. Gray (presenting author), ${ }^{1,2}$ Julian Avila-Pacheco, ${ }^{3}$ David E. Cantonwine, ${ }^{1}$ Thomas F. McElrath, ${ }^{1}$ Clary B. Clish, ${ }^{3}$ and Richa Saxena ${ }^{2,4}$

${ }^{1}$ Division of Maternal-Fetal Medicine, Brigham \& Women's Hospital, Boston, MA; ${ }^{2}$ Center for Genomic Medicine, ${ }^{3}$ Massachusetts General Hospital, Boston, MA; and

${ }^{4}$ Metabolomics Platform and Program in Medical and Population Genetics, Broad Institute, Cambridge, MA

Background and Objectives: As alterations in kidney physiology are fundamentally involved in maternal adaptation to pregnancy, we sought to comprehensively define changes in urinary metabolites across gestation.

Methods: Maternal 1st (T1; 12 weeks) and 2nd (T2; 26 weeks) trimester urine samples from 63 healthy women were obtained from the Brigham \& Women's Hospital LIFECODES cohort. Global metabolic profiling was performed using three well-characterized liquid-chromatography mass spectroscopy (LC-MS) metabolic profiling methods, which identified 351 known metabolites. Data was normalized, $\log 2$ transformed, and features with $>90 \%$ missing values removed. Paired t-tests were performed to assess metabolite differences at each time point and corrected for multiple testing.

Supervised data modeling with partial least squares discriminant analysis (PLS-DA) was performed.

Results: Of 351 known metabolites, 98 were significantly different $(\mathrm{p}<0.01)$ between T1 and T2. PLS-DA clearly distinguished T1 from T2. Of the top 30 metabolites, 18 increased and
12 decreased from T1 to T2. Pathways highlighted by these results include polyamine metabolism, mammary gland proliferation, eicosanoid and fatty acid metabolism, and amino acid metabolism. These pathways are essential for maternal adaptation and fetal development during pregnancy, as they are integral in cellular proliferation/signaling, cell membrane formation, breast milk production, gene transcription/ translation, and protein synthesis.

Conclusions: Global urinary metabolic profiling using LCMS provides a robust platform to understand metabolic changes during normal pregnancy and highlights pathways and features for further investigation. Comprehensive understanding of the urinary metabolome across normal gestation will allow for future investigations of metabolic alterations predisposing to adverse pregnancy outcomes.

\section{P-11: Financial Burden Related to Decisions for Breast Cancer Surgery}

Rachel A. Greenup (presenting author), ${ }^{1,2}$ Christel Rushing, ${ }^{2,3}$ Laura Fish, ${ }^{2,4}$ Brittany M. Campbell, ${ }^{5}$ Lisa Tolnitch, ${ }^{1}$ Terry Hyslop, ${ }^{2,3}$ Jeffrey Peppercorn, ${ }^{6}$ Stephanie Wheeler, ${ }^{7}$ Yousuf Zafar, ${ }^{8}$ Evan R. Myers, ${ }^{9}$ and E. Shelley Hwang ${ }^{1,2}$

${ }^{1}$ Department of Surgery, Duke University; ${ }^{2}$ Duke Cancer Institute, Duke University; ${ }^{3}$ Department of Biostatistics and Bioinformatics, Duke University; ${ }^{4}$ Duke Cancer Control and Population Sciences, Duke University; ${ }^{5}$ Duke School of Medicine, Duke University; ${ }^{6}$ Department of Medicine, Massachusetts General Hospital; ${ }^{7}$ Gillings School of Public Health, University of North Carolina at Chapel Hill; ${ }^{8}$ Department of Medicine, Duke University; and ${ }^{9}$ Department of Obstetrics and Gynecology, Duke University

Background: High cancer treatment costs are increasingly being shifted to patients receiving care, and financial toxicity has been associated with early mortality. Little is known about the risk of financial burden as eligible women choose between equally effective surgical treatments for breast cancer.

Objective: To evaluate financial burden after decisions for breast cancer surgery.

Methods: Female volunteers $\geq 18$ years old with a history of breast cancer were recruited to complete an 88-question electronic survey based on validated or previously published items. Descriptive statistics, hypothesis testing, and regression analysis were used.

Results: In total, 607 women with stage 0-III breast cancer who underwent surgical treatment were analyzed. Median age at diagnosis was 49.6 years old. Median time from diagnosis was 6.7 years (range $0.1-37.1$ ). The majority had private $(70 \%)$ or Medicare (25\%) insurance. Overall, $43 \%$ underwent lumpectomy, $25 \%$ mastectomy, $32 \%$ bilateral mastectomy; $36 \%$ had breast reconstruction. Bilateral mastectomy without and with reconstruction was associated with higher reported incurred debt $(\mathrm{p}<0.01$ and $\mathrm{p}=0.04$ respectively), financial burden $(p=0.02, p<0.01$ respectively), cancer-care induced financial hardship ( $\mathrm{p}<0.01$ for both), and reduction in employment $(\mathrm{p}<0.01, \mathrm{p}=0.02$ respectively) when compared to lumpectomy. Participants who underwent mastectomy with reconstruction also reported higher rates of employment reduction compared to those who underwent lumpectomy $(\mathrm{p}=0.01)$.

Conclusions: The risk of financial burden differs by surgery type with bilateral mastectomy being associated with higher incurred debt, financial burden, and altered employment. Cost 
transparency may improve the quality of preference-sensitive treatment decisions and reduce the risk of financial harm.

\section{P-12: BRCA Testing and Testing Results Among Women 18-65 Years Old}

Fangjian Guo (presenting author), ${ }^{1,2}$ Erika L. Fuchs, ${ }^{1,2}$ Abbey B. Berenson, ${ }^{1,2}$ and Yong-Fang Kuo ${ }^{2,3,4}$

${ }^{1}$ Department of Obstetrics \& Gynecology; ${ }^{2}$ Center for Interdisciplinary Research in Women's Health; ${ }^{3}$ Office of Biostatistics, Department of Preventive Medicine and Community Health; and ${ }^{4}$ Institute for Translational Science, University of Texas Medical Branch, Galveston, TX

Background: BRCA1/2 mutations were discovered in the 1990s in breast or ovarian cancer patients. Since then, genetic testing has been recommended for targeted, individualized cancer prevention and treatment.

Objectives: To assess the trends in BRCA test rates and results among adult women in the U.S.

Methods: Using Clinformatics Data Mart (CDM) Electronic Health Records, we included 223,211 women $18-65$ years old with BRCA testing results from 1/1/2007-9/1/2017. Annual percentage change (APC) in BRCA testing and test results was calculated. Positive test results indicate a high risk for developing susceptible cancers.

Results: Test rates increased significantly from 3.4 per 10,000 women in 2007 to 48.8 per 10,000 women in 2016 (APC 30.8, 95\% confidence interval 26.6-35.1). Positive results decreased from $86.1 \%$ in 2007 to $78.0 \%$ in 2017 (APC $0.6, p=0.1)$. From 2007-2017, decreasing trends in positive results were observed among all three age groups (18-39, 4054 , and 55-65 years; largest in 40-54 group). Among patients with breast or ovarian cancer, positive results decreased from $85.3 \%$ to $62.4 \%$, comparing to among those without breast or ovarian cancer that decreased from $86.2 \%$ to $80.9 \%$ (APC -3.2 vs. $-0.1, p<0.001$ ). In 2015-2017, women with positive test results were less likely to be cancer patients or non-Hispanic Whites, or live in the Northeast or an area with average household income $\geq \$ 50,000$.

Conclusions: There was increasing use of BRCA testing for cancer prevention and treatment. In women with breast or ovarian cancer, the significantly decreasing positive rate indicates a growing loosened criteria for testing subject selection.

\section{P-13: Sex Differences in Chronic Optogenetic Spreading Depression as an Animal Model for Chronic Migraine}

Andrea M. Harriott, ${ }^{1,2}$ and Cenk Ayata ${ }^{1}$

${ }^{1}$ Massachusetts General Hospital, Vascular Neurology Division; and ${ }^{2}$ Headache and Neuropathic Pain Unit, $14913^{\text {th }}$ Street, Neurovascular Lab, Navy Yard, Massachusetts General Hospital, Boston, MA

Background: Chronic migraine is an incapacitating disorder more common in women. There is a dearth of preclinical studies examining the influence of sex and hormones on mechanisms of chronic migraine.

Objective: We used a minimally invasive optogenetic method of spreading depression (SD) to study the impact of sex on mechanisms of chronic migraine.
Methods: SD was elicited in heterozygous optogenetic male and female mice expressing light activated channel rhodopsin-2 using blue wavelength transcranial light stimulation every other day for 14 days. Differences in SD susceptibility, anxiety and mechanical pain related behaviors were examined using ANOVA and test.

Results: Chronic SD decreases SD susceptibility. The threshold for SD activation increases from $6.8 \pm 2.4 \mathrm{~mW}$ to $8.7 \pm 0.58 \mathrm{~mW}(\mathrm{p}=0.016)$. There were no sex differences in SD threshold. While there appear to be sex differences in the SD waveform, there were no differences in latency. Chronic SD increased time spent in the outer perimeter of an open field (thigmotaxis). The fraction of time spent in the outer perimeter was $0.8 \pm 0.1$ in controls versus $0.93 \pm 0.05$ for chronic SD $(\mathrm{p}=0.042)$. Both males and females demonstrated similar increases in anxiety. Chronic SD decreased periorbital mechanical thresholds, control $0.142 \pm 0.04 \mathrm{~g}$, chronic SD $0.49 \pm 0.03$ $(\mathrm{p}<0.001)$. This was observed in both sexes. Chronic SD unexpectedly increased ipsilateral hindpaw mechanical thresholds in females only(control $1.2 \pm 0.28 \mathrm{~g}$, chronic SD $2 \pm 0 \mathrm{~g}$, $\mathrm{p}=0.025$ ).

Conclusions: Chronic SD is associated with reduced cortical excitability which may act to limit the impact of SD, increased anxiety and trigeminal pain related behaviors.

\section{P-14: Intergenerational Effects of Torture and War Trauma: Maternal Caregivers' Perspectives on Family Functioning and Youth Adjustment}

Sarah J. Hoffman (presenting author), ${ }^{1}$ Maria Vukovich, ${ }^{2}$ Abigail Gewirtz, ${ }^{3}$ Joseph Gaugler, ${ }^{4}$ and Cheryl Robertson ${ }^{1}$

${ }^{1}$ School of Nursing, University of Minnesota; ${ }^{2}$ Center for Victims of Torture; ${ }^{3}$ FSoS, University of Minnesota; and ${ }^{4}$ School of Public Health, University of Minnesota

Background: Intergenerational trauma is defined as the passage of trauma experienced by one generation onto members of future generations who were not exposed to the original trauma. An emerging body of evidence suggests that refugee maternal caregivers' experiences of torture bear important impact on health outcomes of youth and families.

Objective: Compare patterns of family functioning and youth adjustment in a sample of Karen refugees where women experienced torture versus war trauma alone. We hypothesize a direct association between family functioning and youth adjustment. Maternal torture exposure and higher perceived severity of traumatic experiences in war will be associated with imbalance in family functioning and youth adjustment in comparison to war trauma and lower perceived severity.

Methods: Within the overarching sequential explanatory mixed methods design, refugee maternal caregivers of youth (11-23 years) will complete quantitative instruments assessing mental health, family adaptability and cohesion, youth adjustment, and war trauma/torture exposure.

Results: Of the total proposed sample of 100 women, we have enrolled 61 participants. Key demographic variables of the sample to date include age $(M=45.6$, range $34-58)$, years in resettlement $(\mathrm{M}=4.9$, range $1-11)$. To investigate predictable relationships among family functioning and youth adjustment we will use multiple regression modeling. Potentially important predictors of differential youth adjustment will be examined in the models. We will complete data collection and analysis for this study phase in July 2018. 
Conclusions: The current investigation will inform the development of a family-centered intervention to interrupt intergenerational trauma between women refugee survivors of torture and their children.

\section{P-15: Trends in Opioid Use Disorder Treatment for Pregnant Women on Medicaid, 2009-2015}

Elizabeth E Krans, ${ }^{1}$ Joo Yeon Kim, ${ }^{2}$ David Kelley, ${ }^{3}$ and Marian Jarlenski (presenting author) ${ }^{2}$

${ }^{1}$ Department of Obstetrics, Gynecology and Reproductive Sciences, University of Pittsburgh School of Medicine and Magee-Womens Research Institute; and ${ }^{2}$ Department of Health Policy and Management, University of Pittsburgh Graduate School of Public Health; and ${ }^{3}$ Department of Human Services, Commonwealth of Pennsylvania

Background: Despite the effectiveness of medication assisted treatment (MAT) among pregnant women with opioid use disorder (OUD), there may be an unmet treatment need in this population.

Objective: To assess trends in utilization of MAT (methadone or buprenorphine) and behavioral health counseling among Medicaid-enrolled pregnant women with OUD.

Methods: Retrospective cohort study using healthcare administrative data for 12,587 women diagnosed with OUD in pregnancy in Pennsylvania Medicaid between 2009-2015. We calculated time trends in prevalence of MAT and behavioral health counseling using multivariable regression to adjust for demographic and clinical factors. Predicted probabilities of receiving methadone, buprenorphine, and behavioral health counseling, with corresponding $95 \%$ confidence intervals, were examined to investigate time trends.

Results: Across all years, $44.1 \%$ of pregnant women had no MAT observed, $27.1 \%$ received buprenorphine, and $28.7 \%$ received methadone. Less than half of women had any behavioral health counseling in pregnancy. The adjusted prevalence of methadone use in pregnancy declined from 31.6\% (95\% CI: $29.3,33.9$ ) in 2009 to $25.2 \%$ (95\% CI: $23.3,27.1)$ in 2015 . The adjusted prevalence of buprenorphine use in pregnancy increased from $15.8 \%(95 \%$ CI: $13.9,17.8)$ to $30.9 \%$ (95\% CI: $28.8,33.0)$. The adjusted number of behavioral health counseling visits in pregnancy was 4.0 (95\% CI: 3.3,4.7) among women with no MAT observed, and 6.4 (95\% CI: 4.9,7.9) among women receiving methadone.

Conclusions: Rates of buprenorphine use in Medicaidenrolled pregnant women with OUD increased significantly over time, with a small concurrent decline in methadone use. Behavioral health counseling use was low, but highest among women receiving methadone.

\section{P-16: De Novo Single Gene Variants Associated with Omphalocele, Exstrophy, Imperforate Anus, Spinal defects (OEIS)}

Angie C. Jelin, ${ }^{1,2}$ Nara Sobreira, ${ }^{2,3}$ Elizabeth Wohler, ${ }^{2}$ Karin Blakemore, ${ }^{1,2}$ Dane Witmer, ${ }^{4}$ Heather Di Carlo, ${ }^{5}$ John Gearhart, ${ }^{5}$ and David Valle ${ }^{2,3}$

${ }^{1}$ Department of Gynecology and Obstetrics, Johns Hopkins School of Medicine; ${ }^{2}$ McKusick-Nathans Institute of Genetic Medicine, Johns Hopkins School of Medicine; ${ }^{3}$ Department of Pediatrics, Johns Hopkins School of Medicine; ${ }^{4}$ Center for
Inherited Disease Research, Johns Hopkins University; and ${ }^{5}$ Department of Pediatric Urology, Johns Hopkins School of Medicine

Background: OEIS is a severe fetal anomaly associated with genital, urinary and spinal defects that occur during early embryogenesis. A heterogenous etiology has been speculated, however the high rate of infertility suggests that de novo single gene mutations could be largely causative. Candidate genes have been proposed based upon one prior sequencing study.

Objective: We sought to identify de novo single gene variants associated with OEIS.

Methods: Pediatric urology patients with OEIS were clinically recruited and consented. Genomic DNA was extracted from the peripheral blood or saliva of trios (probands, mothers and fathers). Whole exome sequencing was performed on exonic and flanking intron regions with the Agilent SureSelect HumanAllExonV5Clinical_S06588914 kit and paired end $125 \mathrm{bp}$ reads with the Illumina HiSeq2500 platform. Variants with an internal or ExAC allele frequency of greater than $1 \%$ were removed, as were those with a depth $<10$.

Results: We identified a total of ten rare de novo single gene mutations in 8 OEIS trios studied. A frameshift mutation was identified in the gene HSD11B1. The remaining mutations were missense single nucleotide variants in EIF6, FANCE, FLCN, INTS2, RPL4, WDR81, TCP11L1, TMEM120B, ZNF280B. One proband had 2 de novo mutations, a second proband had de novo mutations in 3 genes and 1 proband had none. Each mutation was unique to a specific proband.

Conclusions: Our etiologic investigation of OEIS using WES resulted in the identification of several de novo single gene mutations arising in affected probands. Functional investigation of the mutations is necessary to prove causation.

\section{P-17: Moderation-Mediation Effects Between Labor and Postpartum Pain, Prenatal Factors, and Postpartum Depression}

Grace Lim (presenting author), ${ }^{1}$ Lia M. Farrell, ${ }^{1}$ Stephanie Nam, ${ }^{1}$ and Ajay D. Wasan ${ }^{2}$

${ }^{I}$ Department of Anesthesiology, University of Pittsburgh, Magee-Womens Hospital of UPMC; and ${ }^{2}$ Department of Anesthesiology and Psychiatry, University of Pittsburgh, University of Pittsburgh Medical Center

Background: Labor pain has been linked to postpartum depression, but the mechanism for this relationship is unclear. Moderator-mediator models can investigate indirect associations between pain and depression. The nature of the relationship between labor and puerperal pain and depression is critical to understanding modifiable risk factors for depression.

Objectives: To assess psychometric and psychological factors predictive of labor and puerperal pain and depression, and to assess moderating-mediating effects between pain and depression.

Methods: A prospective observational design was chosen. Primiparous women planning a vaginal delivery were enrolled in the third trimester and followed until 3 months postpartum. The primary outcome was depression score at 6 weeks postpartum (Edinburgh Postnatal Depression Scale, EPDS). Baseline assessments of depression, anxiety, perceived social support, catastrophizing, and quantitative sensory testing (QST) variables were completed. During labor and delivery, hourly assessments 
of pain intensity and pain unpleasantness using an electronic pain diary were recorded. Postpartum assessments included EPDS, pain inventory, anxiety, perceived stress, maternal-infant bonding, breastfeeding, infant development, and parenting selfefficacy. Analyses will be stratified by epidural analgesia utilization, to assess a moderation effect by analgesia preference. Multivariable linear regression will assess: (1) the pain and depression relationship, controlling for covariates; and (2) pain as a mediator in the relationship between psychiatric variables and depression.

Results: 57 women who received labor epidural analgesia and 17 women who received no analgesia completed study procedures. Analysis is in progress.

Conclusions: Factors associated with labor and puerperal pain and depression will be identified.

Moderator-mediator effects will be presented.

\section{P-18: Improvement in Metabolism in Obese Postmenopausal Women with Novel Estrogen Complexes}

Dragana Lovre (presenting author), ${ }^{1}$ Bonnie Katalenich, ${ }^{2}$ Erin Peacock, ${ }^{3}$ Sarah B. Camitta, ${ }^{4}$ Vivian Fonseca, ${ }^{1}$ and Franck Mauvais-Jarvis ${ }^{1}$

${ }^{1}$ Department of Medicine, Division of Endocrinology and Metabolism, Tulane School of Medicine; ${ }^{2}$ Louisiana Clinical \& Translational Science Center, Tulane School of Medicine; ${ }^{3}$ Department of Medicine, Tulane School of Medicine; and ${ }^{4}$ Department of Global Community Health and Behavioral Sciences, Tulane School of Public Health and Tropical Medicine, New Orleans, LA

Background and Objectives: Due to increasing life expectancy, women will spend almost half of their lives in menopause, which may increase risk for metabolic syndrome (MetS) and type 2 diabetes (T2D). Randomized controlled trials show that menopause hormone therapy (MHT) reduces the incidence of T2D. A novel menopausal therapy, approved in 2013 by the FDA, combines conjugated estrogen (CE) and a tissue-selective estrogen complex bazedoxifene (BZA). In a mouse model, CE/ BZA prevents estrogen deficiency-induced obesity, and T2D, as efficiently as $\mathrm{CE}$ alone, but without causing endometrial hyperplasia. Our objective is to assess the effect of CE/BZA vs. placebo on glucose homeostasis and body composition in obese post-menopausal women. Our hypothesis is that CE/BZA will improve obesity, insulin resistance, systemic inflammation, and endothelial dysfunction in obese postmenopausal women.

Methods: We conducted a pilot randomized double blind placebo-controlled trial to test our hypothesis. Obese postmenopausal women were recruited and randomly assigned to 12 weeks of treatment with CE/BZA or placebo. At baseline and after 12 weeks, we measured peripheral arterial tone, IV-glucose tolerance test, DXA scan, and inflammatory biomarkers to assess endothelial dysfunction, glucose homeostasis, body composition and inflammation, respectively. Anthropometric measurements were assessed at baseline and at 4 weeks, 8 weeks, and 12 weeks.

Results: Twelve obese postmenopausal females (mean age $55.1 \pm 1.9$ years, African-American 16.6\%, BMI 36.3 \pm 6.3 , years since LMP $3.3 \pm 3.0$ ) were enrolled. Data collection has been completed and analysis is underway.

Conclusions: This research will increase our understanding of the effects of CE/BZA on metabolic dysfunction in obese postmenopausal women.
P-19: Associations of Self-Reported Polycystic Ovary Syndrome (PCOS), Irregular Menstrual Cycles, and the Metabolic Syndrome in Premenopausal Hispanic/Latina Women in the Hispanic Community Health Study/Study of Latinos (HCHS/SOL)

Michelle L. Meyer, ${ }^{1}$ Daniela Sotres-Alvarez, ${ }^{2}$ Anne Steiner, ${ }^{3}$ Larry Cousins, ${ }^{4}$ Gregory A. Talavera, ${ }^{5}$ Jianwen Cai, ${ }^{2}$ Martha L. Daviglus, ${ }^{6}$ and Laura R. Loehr ${ }^{7}$

${ }^{1}$ Department of Emergency Medicine, University of North Carolina-Chapel Hill, Chapel Hill, NC; ${ }^{2}$ Department of Biostatistics, University of North Carolina-Chapel Hill, Chapel Hill, NC; ${ }^{3}$ Department of Obstetrics and Gynecology, University of North Carolina-Chapel Hill, Chapel Hill, NC; ${ }^{4}$ Children's Specialists of San Diego, San Diego, CA; ${ }^{5}$ Division of Health Promotion and Behavioral Science, Graduate School of Public Health, San Diego State University, San Diego, CA; ${ }^{6}$ Institute for Minority Health Research, University of Illinois at Chicago College of Medicine, Chicago, IL; and ${ }^{7}$ Department of Epidemiology, University of North Carolina-Chapel Hill,

Chapel Hill, NC

Background: Irregular menstrual cycles are a sign of polycystic ovary syndrome (PCOS) in women, which is associated with cardiometabolic risk factors; however, the characterization is incomplete in premenopausal women and high-risk groups such as Hispanic/Latina women.

Objective: To estimate the association of self-reported PCOS, irregular menstrual cycles, and the metabolic syndrome (MetS) in premenopausal Hispanic/Latina women.

Methods: This cross-sectional analysis includes 1,524 women $<44$ years old from visit 2 (2014-2017; mean age 34 years) of HCHS/SOL, a U.S. community-based cohort study of Hispanic/ Latino adults. Women were asked if they had PCOS and to specify their menstrual cycle length at age 20-40 when not taking birth control pills or hormones. Cycles were defined as $<24$ days, 24-35 days (referent), >35 days, and irregular. We defined the MetS using the NCEP-ATPIII criteria and calculated odds ratios (OR) and 95\% confidence intervals (CI) adjusted for complex survey study design, demographics, and body mass index.

Results: Overall, $6.1 \%$ self-reported PCOS, $2.9 \%$ had menstrual cycles $>35$ days, and $15.4 \%$ had irregular menstrual cycles. Self-reported PCOS was associated with a 1.8 (CI: 0.9-3.5) times higher odds of the MetS compared to those without self-reported PCOS, although not statistically significant. Women reporting irregular menstrual cycles had a 1.8 (CI: 1.1-2.8) times higher odds of the MetS compared with women reporting cycles 24-25 days.

Conclusions: Irregular menstrual cycles were associated with the MetS and could indicate risk of metabolic disease. Measurement of androgens and identification of PCOS will be critical to characterize these relationships.

\section{P-20: Communication Differences in Women after Right Brain Stroke}

Jamila M. Minga (presenting author), ${ }^{1,2}$ Eugene Oddone, ${ }^{3}$ Davida Fromm, ${ }^{4}$ and Adam Jacks ${ }^{5}$

${ }^{1}$ Communication Disorders Program, North Carolina Central University; ${ }^{2}$ Department of Obstetrics and Gynecology, Duke University; ${ }^{3}$ Department of Veteran's Affairs, Duke University; ${ }^{4}$ Department of Psychology, Carnegie Mellon University; and ${ }^{5}$ Department of Allied Health Sciences, University of North Carolina at Chapel Hill 
Background: Right hemisphere strokes (RHS) can result in lasting, yet under-identified, communication impairments. Left hemisphere stroke affects speech fluency and word finding, but RHS affects adhering to conversational norms and rules that can result in inadequate social interactions and negative socioeconomic outcomes. This may be observed in patterns of questionasking, a crucial component of sustaining conversations.

Objective: To determine if women with RHS use questions differently than neurologically-healthy women (NHW).

Methods: Women in the chronic phase of RHS recovery and NHW were recruited through stroke support groups and social media. Each posed questions during a face-to-face videorecorded session for two different tasks: 1) getting to know an unfamiliar person and 2) determining the purpose of nine unfamiliar objects. No time restrictions were imposed, but 10 questions per object were requested. Questions were transcribed, counted, and coded for question type (polar [e.g. yes/no], content) using part of an established coding scheme.

Results: Twelve age-matched (mean \pm SD: RHS: $48 \pm 2.6$ and NHW:49 \pm 2.8 years) women participated in this pilot study (6/ group). Women with RHS posed 473 total questions, $1 \%$ fewer than NHW (503). For the person and object tasks, RHS women posed fewer polar questions (16\% and $15 \%$, respectively) and more content questions (14\% and 17\% respectively) than NHW.

Conclusions: These findings suggest that right hemisphere stroke may contribute to differences in question-asking. If confirmed in larger sample sizes, question-patterns may be a promising diagnostic indicator of communication deficits following RHS and the basis for new assessment and treatment protocols.

\section{P-21: Evaluating Electronic Cognitive Behavioral Therapy to Reduce Insomnia, Sleep Aid Use, and Stress in Appalachian Women Ages 45+}

Mairead Eastin Moloney

Department of Sociology, University of Kentucky

Background: Sedative hypnotics $(\mathrm{SH})$ pose myriad health risks (e.g., falls, cancer, neurocognitive disorders) and may be over-prescribed as a treatment for stress-induced, transient insomnia. Unfortunately, accessible, effective, non-drug alternatives are rarely employed, particularly in high-risk populations such as Appalachian women (AW). AW experience multiple economic and psycho-social stressors, have high rates of insomnia and SH use, and fragmented healthcare access.

Objective(s): This innovative pilot project assesses feasibility, acceptability, and effectiveness of SHUTi (Sleep Healthy Using the Internet), a well-validated, online cognitive behavioral therapy for insomnia program in AW ages $45+(\mathrm{N}=47)$.

Methods: Using a mixed-methods approach this pre-post intervention evaluation will determine the extent to which SHUTI reduces sleeplessness, stress, and $\mathrm{SH}$ use. Participants are AW ages $45+$ with Internet access who experience insomnia (3+ months) and use SH. SHUTi is comprised of 6, once-weekly online educational sessions ( $\sim 40-60$ minutes) and a daily online sleep diary ( $\sim 2-3$ minutes). Pre- and post- intervention participants complete an online survey and semi-structured qualitative interview. We will use grounded theory to analyze qualitative data. Multi-level modeling will be used to assess changes over time in quantitative data. We hypothesize that SHUTi will increase sleep latency while decreasing stress and SH use.

Results: Forty-seven women enrolled. Eight were lost to attrition. Thus far, 36 have completed the SHUTi program. Pre- liminary analyses on the first 18 completers indicate statistically significant improvements in sleep and stress, and reduced $\mathrm{SH}$ use. Analyses are ongoing.

Conclusions: Preliminary analyses suggest SHUTi feasibility, acceptability and effectiveness in this group of high-risk women.

\section{P-22: Protons Activate ATR Pathway Signaling and Increase Dependence on BRCA2}

Qin Zhou, ${ }^{1}$ Michelle E. Howard, ${ }^{1}$ Jann N. Sarkaria, ${ }^{1}$ Zhenkun Lou, ${ }^{2}$ Michael G. Herman, ${ }^{1}$ Chris Beltran, ${ }^{1}$ and Robert W. Mutter (presenting author) ${ }^{1}$

\section{${ }^{1}$ Department of Radiation Oncology; and ${ }^{2}$ Department of Oncology, Mayo Clinic, Rochester, Minnesota}

Background: Proton therapy (PT) is under investigation in breast cancer due to physical properties that enable rapid dose fall off beyond the target, limiting exposure to normal tissues. Mutations in BRCA2 increase the risk of breast cancer and other malignancies but also render tumor cells uniquely sensitive to some forms of DNA damage.

Objectives: To investigate differences in DNA damage signaling between PT and photons and to correlate linear energy transfer (LET), which increases at the end of proton range, with RBE in BRCA2 and wild-type isogenic cells.

Methods: BT549 and MDA-MB-231 breast cancer cells and BRCA2 wild-type (V79) and mutant (VC8) Chinese hamster cells were irradiated with $6 \mathrm{MV}$ photons and $71 \mathrm{MeV}$ protons at entrance $(\mathrm{LETd}=1.78 \mathrm{keV} / \mathrm{um})$ or at the Bragg peak (BP, LETd $=4.35 \mathrm{keV} / \mathrm{um}$ ) and DNA damage signaling and cell survival was assessed using immunoblot, immunofluorescence, and clonogenic assays. RBE values were calculated by comparing the physical dose (D6MV/Dp) that results in 50\% (RRRRRR0.5) and $10 \%$ (RRRRRR0.1) cell survival.

Results: The proton RBE was slightly greater for VC8 relative to V79 at entrance. At $50 \%$ and $10 \%$ survival, the RBEs were $1.39 \pm 0.26$ and $1.14 \pm 0.07$ vs $1.05 \pm 0.17$ and $1.09 \pm 0.08$ for VC8 and V79, respectively. At the BP, the proton RBE increased markedly in VC8 relative V79. At 50\% and $10 \%$ survival, the RBE values were $2.06 \pm 0.41$ and $1.67 \pm 0.11 \mathrm{vs}$ $1.33 \pm 0.19$ and $1.29 \pm 0.07$ for VC8 and V79, respectively. Compared with entrance or photons, PT at the BP resulted in increased p-RPA, p-ATR, p-Chk1 and $\gamma \mathrm{H} 2 \mathrm{AX}$ at early and late timepoints.

Conclusions: High LET PT DNA activates ATR pathway signaling. This DNA damage is more dependent on BRCA2mediated repair.

\section{P-23: Individualized Versus Standard Post-Discharge Opioid Prescribing Following Cesarean Birth: A Randomized Controlled Trial}

Sarah S. Osmundson SS (presenting author), ${ }^{1}$ Britany L. Raymond, ${ }^{2}$ Bradley T. Kook, ${ }^{2}$ LeAnn Lam, ${ }^{3}$ Elizabeth B. Thompson, ${ }^{4}$ Leslie A. Schornack, ${ }^{2}$ Catherine E. Voorhees, ${ }^{1}$ and Michael G. Richardson ${ }^{2}$

${ }^{1}$ Department of Obstetrics and Gynecology, Vanderbilt University Medical Center Nashville, TN; ${ }^{2}$ Department of Anesthesiology Vanderbilt University Medical Center Nashville, TN; and ${ }^{3}$ University of Utah, Salt Lake City, Utah; ${ }^{4}$ Vanderbilt University 
Background and Objectives: Overprescribing is a major source of opioids available for misuse and diversion. This study evaluates whether individualized opioid prescribing reduces the number of unused opioid tablets after cesarean.

Methods and Results: We conducted a randomized, controlled trial of women $\geq 18$ years undergoing cesarean. Participants were randomized at discharge in to a standard (30 tablets of 5mg oxycodone) vs individualized opioid prescription (predicted based on each patient's inpatient opioid use). All women were contacted starting 14 days after cesarean to assess number of opioid tablets used and adequacy of pain control. The primary outcome was number of unused opioid tablets. Between 6/14/ 2017 and 8/25/2017, we screened 323 women and randomized 172 participants. Baseline characteristics and inpatient opioid use were similar between groups. Women in the individualized group had $50 \%$ fewer unused tablets than women in the standard arm (5 [IQR 1-8] vs 10 [IQR 0-22], p<0.001). There were no differences between the standard and individualized groups in the proportion of women who used no opioids or all opioids, and no difference in the proportion of dispensed opioids used $(60 \%$ [IQR 23-100] vs 61\% [29-89], $\mathrm{p}=.93$ ). Women in the individualized group used only half the number of prescribed opioids as women in the standard group. Patient reported pain outcomes did not differ significantly by group.

Conclusions: Individualized opioid prescriptions based on inpatient use reduces both the amount of opioid used and unused after discharge compared to standard prescribing.

\section{P-24: HTRA1 is a Novel Regulator of Angiogenesis in Maternal Pre-Eclampsia and Preterm Infant Retinopathy of Prematurity}

Leah A Owen (presenting author), ${ }^{1}$ Samuel A. Collazo, ${ }^{3}$ Blair Wood, ${ }^{1}$ Mark J. Cody, ${ }^{2}$ Margaret M. DeAngelis, ${ }^{1}$ and Christian C. Yost ${ }^{2}$

${ }^{1}$ Department of Ophthalmology and Visual Sciences, University of Utah School of Medicine. Salt Lake City, UT;

${ }^{2}$ Division of Neonatology, Department of Pediatrics, University of Utah School of Medicine, Salt Lake City, UT; and ${ }^{3}$ School of Medicine, University of Puerto Rico, Medical Sciences Campus

Background: Maternal pre-eclampsia and preterm infant retinopathy of prematurity (ROP) are characterized by aberrant angiogenesis. The molecular pathophysiology for each condition is unclear, as is the degree to which these pathologies display overlapping mechanisms as has been suggested in epidemiologic literature. HTRA1, a secreted serine protease differentially expressed in maternal pre-eclampsia and neovascular retinal degeneration, is implicated in angiogenesis via TGF $\beta-1$ signaling.

Objective: We hypothesized that systemic HTRA1 and the downstream angiogenic mediator TGF $\beta-1$ are similarly dysregulated in patients with maternal preeclampsia and ROP, representing a novel oxygen responsive angiogenic pathway.

Methods: We analyzed systemic HTRA 1 and TGF $\beta$ - 1 levels in preterm infants $(n=25)$ as well as women who experienced preterm birth $(n=25)$ using ELISA. Data were stratified by maternal pre-eclampsia and infant ROP status. In vitro analysis measured HTRA1 protein expression following ocular cell growth at varying oxygen tensions using ELISA.

Results: We found that HTRA1 is decreased in preterm infants with ROP as well as in the systemic maternal circulation of women who delivered preterm in the setting of pre-eclampsia.
In both disease states this correlated with increased systemic TGF $\beta$-1 expression as well as increased placental TGF $\beta-1$. In vitro, retinal pigment epithelial cells show decreased HTRA1 protein expression over time with elevated oxygen tension, in a dose dependent fashion.

Conclusions: HTRA 1 and TGF $\beta-1$ are similarly dysregulated in maternal pre-eclampsia and ROP and this dysregulation may be mediated by oxygen. These data support that in other fields implicating HTRA1 as a novel angiogenic mediator in disease.

\section{P-25: Metabolic Effects of Sugar-Sweetened Beverage Consumption in African-American Versus White Women}

Candice Allister Price, ${ }^{1}$ Valentina Medici, ${ }^{2}$ Vivien Lee, ${ }^{1}$ Marinelle V. Nunez, ${ }^{1}$ Nancy L. Keim, ${ }^{3}$ and Kimber L. Stanhope ${ }^{1}$

${ }^{1}$ From the Department of Molecular Biosciences, School of Veterinary Medicine (CAP, KLS, VL) the Department of Nutrition (KLS, MVN, NLK); ${ }^{2}$ The Division of Gastroenterology and Hepatology, School of Medicine (VM); and ${ }^{3}$ U.S.

Department of Agriculture, Western Human Nutrition Research Center, Davis, CA (NLK)

Background: Sugar-sweetened beverages (SSB) are associated with increased cardiovascular disease (CVD)- and type 2 diabetes (T2D)- in adults $(1,2)$. However, it is unclear if these metabolic effects of SSB apply to African-American (AA) women, a group reported to be the highest consumers of SSB (3), who have higher prevalences of CVD and T2D (4) and generally have a paradoxically healthier CVD phenotype [lower triglycerides and low density lipoprotein (LDL), and higher high density lipoprotein (HDL) levels, all markers of lower CVD risk] compared to white women.

Objective: To determine if SSB consumption has similar effects on cardiometabolic risk factors in AA and white women.

Methods: Healthy AA $(n=7)$ and white $(n=7)$ women (mean BMI $30.4 \pm 3.4 \mathrm{~kg} / \mathrm{m}^{2}$; mean age $30.1 \pm 6.0$ years) consumed SSBs provided at $25 \%$ of their daily energy-requirement for 2 weeks.

Results: Preliminary results suggest that 2 weeks of SSB consumption in AA women resulted in greater increases in OGTT glucose $(\mathrm{p}<0.05)$ and greater reductions in insulin sensitivity $(\mathrm{p}=0.08)$ compared to white women. In contrast, total cholesterol $(\mathrm{p}=0.25)$ and HDL $(\mathrm{p}=0.34)$ were unaffected in AA women, whereas LDL, ApoB, total cholesterol and nonHDL cholesterol [total cholesterol minus HDL] were all significantly increased by SSB in white women and were overall greater than those values in AA women.

Conclusions: These interim results of an ongoing study, show differential metabolic changes in response to SSB consumption between AA and white women. The underlying mechanisms require further investigation at the molecular level, work that is ongoing.

\section{P-26: Psychometric Development and Validation of an Instrument to Prospectively Measure Contraceptive Sexual Acceptability}

Jessica N. Sanders (presenting author), ${ }^{1}$ Daniel E. Adkins, ${ }^{2}$ Kelsey Q. Wright, ${ }^{3}$ David K. Turok, ${ }^{1}$ and Jenny A. Higgins ${ }^{3,4}$

${ }^{1}$ Department of Obstetrics and Gynecology, University of Utah; ${ }^{2}$ Department of Sociology, University of Utah; ${ }^{3}$ Department of 
Obstetrics and Gynecology University of Wisconsin-Madison; and ${ }^{4}$ Department of Gender Studies, University of Wisconsin-Madison

Background and Objectives: Clinical contraceptive care overwhelmingly prioritizes efficacy and often neglects key domains of user acceptability, including sexual experience. Existing approaches to measuring contraceptive sexual acceptability suffer important limitations. Preliminary work demonstrates how sexuality impacts choice and use of contraceptive methods over time; however, robust sexuality measures specific to the contraceptive context are needed. The objective of this work is to psychometrically develop and validate an instrument to prospectively measure Contraceptive Sexual Acceptability (CSA).

Methods: Our previous work defined the construct of sexual acceptability and informed the use of items to assess changes in sexual experiences in relation to an individual's contraceptive use. We incorporated these items in the HER Salt Lake longitudinal cohort study $(n=4,427)$. Participants completed surveys at baseline, $1,3,6$, and 12 months. We used factor analysis (exploratory and confirmatory) to assess underlying structure of the items to optimize measurement validity and reliability of the sexual acceptability latent factor/s. After identifying the best fitting models of the sexual acceptability factors, we test the association of these factors to key outcomes including method choice, satisfaction and continuation.

Results: When assessing properties of prospective CSAmenstrual/hormonal, self-responsiveness, physical (lubrication, arousal, pain), partner factors, and orgasm domains emerged. Reliability, Internal Structure Validity, Differential Functioning will be presented.

Conclusions: Development of a robust tool incorporating the identified factors will support patient- centered contraceptive care. Future efforts should integrate the Contraceptive Sexual Acceptability Measure into research, clinical care and contraceptive development of new methods with high user acceptability.

\section{P-27: Sex-Based Differences in Knee Ligament Strain Prior To and Following Initial Contact During Cadaveric Landing Simulations}

Nathan D. Schilaty (presenting author), Nathaniel A. Bates, Aaron J. Krych, and Timothy E. Hewett

Department of Orthopedic Surgery, Mayo Clinic, Rochester, $M N$

Background and Objectives: Females have an increased risk of injury to the anterior cruciate ligament (ACL) compared to male counterparts. $75 \%$ of ACL injuries occur by non-contact mechanisms with landing, cutting, or pivoting maneuvers. This study was designed to determine whether males and females differ in ligament strain loading prior to and after initial contact during a common athletic task - a drop vertical jump (DVJ). We hypothesized that female limbs would demonstrate increased ACL ligament strain that predispose females to ACL injury compared to males.

Methods: A custom-designed cadaveric impactor simulated DVJs in lower extremities of 36 specimens (18M:18F; 40.9 \pm 8.4 years). Pneumatic cylinders applied randomized in vivo measured knee forces/moments to the tibia and a gravity-driven drop sled of 0.5 body weight was released from a height of $31 \mathrm{~cm}$ with quadriceps and hamstrings co-contracted (1:1 ratio). Differential Variable Reluctance Transducers measured ligament strain. We analyzed three risk category conditions (low, med, high).

Results: Females demonstrated significant increases of ACL strain $(\mathrm{t} 98=2.827 ; \mathrm{p}=0.006)$ prior to and following initial contact $(\mathrm{t} 98=2.559 ; \mathrm{p}=0.012)$ compared to males. Prior to and following initial contact, females had $1 \%$ and $2.7 \%$ increased strain than males, respectively. There were no significant differences of medial collateral ligament (MCL) strain prior to or following initial contact between sexes, $(\mathrm{t} 98=-0.961 ; \mathrm{p}=0.339)$ and $(\mathrm{t} 98=1.530 ; \mathrm{p}=0.129)$, respectively.

Conclusions: Females exhibit increased ACL strain with similar loading conditions than males that likely predispose them to ACL injury compared to males. The same conditions did not exhibit any differences between sexes for MCL strain.

\section{P-28: Sex-based Analysis in Mouse Model of Exudative Age-related Macular Degeneration in the Presence of Systemic Inflammation by Collagen-Induced Arthritis}

Gloriane Schnabolk (presenting author) ${ }^{1}$ Elisabeth Obert, ${ }^{1}$ Nirmal K. Banda, ${ }^{2}$ and Bärbel Rohrer ${ }^{1,3}$

${ }^{1}$ Department of Ophthalmology, Medical University of South Carolina, Charleston, SC; ${ }^{2}$ Division of Rheumatology, Department of Medicine, University of Colorado Anschutz Medical Campus, Aurora, CO; and ${ }^{3}$ Ralph H. Johnson VA Medical Center, Division of Research, Charleston, SC

Background and Objectives: Age-related macular degeneration, the leading cause of blindness in the USA, is characterized by inflammation and increased female incidence. While rheumatoid arthritis (RA), diagnosed predominantly in women, shares a similar inflammatory profile, little is known about its possible interaction with AMD. This study investigates the association between RA and AMD, in mice of both sexes.

Methods: A mouse model of collagen induced arthritis (CIA) followed by laser-induced choroidal neovascularizaion (CNV), a model for exudative AMD, was implemented in male and female C57BL/6J mice. CNV lesion size was quantified using optical coherence tomography, and function assessed using optokinetic response and electroretinography c-wave analysis.

Results: CNV size was reduced in the presence of CIA in both sexes (control: $8530 \pm 496.3 \mu \mathrm{m} 2$; CIA: $5961 \pm 481.9 \mu \mathrm{m} 2$; $\mathrm{p} \leq 0.001)$. Optokinetic response was decreased in both spatial acuity (control: $0.37 \pm 0.009$; CIA: $0.32 \pm 0.011: \mathrm{p} \leq 0.01$ ) and contrast sensitivity (control: $5.85 \pm 0.63$; CIA: $2.99 \pm 0.38$; $\mathrm{p} \leq 0.001$ ) in the presence of CIA. No significant sex-based difference was observed for optical coherence tomography and optokinetic response. C-wave analysis indicated a significant increase in response related to female sex $(p<0.05)$ but not CIA.

Conclusions: Despite sharing a similar inflammatory profile, CIA and CNV are not additive.

Reduced spatial acuity and contrast sensitivity by CIA suggest decreased ocular and CNS function in the presence of systemic inflammation. As there was a significant difference in retinal pigment epithelium c-wave response between sexes, further sexbased analyses are underway.

\section{P-29: Ocular Complications in in Pediatric Ebola Survivors}

Jessica G. Shantha (presenting author), ${ }^{1}$ Natalie Weil, ${ }^{1}$ Chris Miller, ${ }^{1}$ Gilberte Bastien, ${ }^{2}$ and Steven Yeh $^{1}$

${ }^{1}$ Department of Ophthalmology, Emory University; and
${ }^{2}$ Department of Psychology, Morehouse School of Medicine

Background: Ebola virus disease (EVD) survivors are at risk for post-EVD sequelae, which include uveitis in approximately 
$13-34 \%$. It is unknown the extent to which pediatric patients develop ocular complications. In pediatric patients this has the potential for lifelong morbidity and quality-of-life reduction associated with untreated vision impairment.

Objective: Determine whether prior EVD infection is associated with eye disease including uveitis in a pediatric population compared to a close-contact cohort of EVD survivors.

Methods: A cross-sectional pilot study was performed on pediatric ( $<18$ years) EVD survivors and close contacts. Demographics, ocular and medical history were collected, and an ophthalmic exam performed. Laboratory investigations were EBOV IgG to verify survivor status. Independent variables potentially contributing to ophthalmic diagnosis included EVD survivor status and female sex.

Results: Median age was 10 years (range 1.6-18 years) and $43 \%$ were female. 23 EVD survivors and 63 close-contacts were evaluated. 19 patients tested positive for Ebola IgG. Ocular diseases observed included refractive error $(15,17 \%)$, vernal keratoconjunctivitis $(11,13 \%)$, motility disorders $(7,8 \%)$ and uveitis $(3,3 \%)$. There were no statistically significant differences between ophthalmic diagnoses when comparing EVD survivors to close contacts (Fishers, p-value $>0.05$ ). When comparing sex differences between disease development the only statistically significant difference was refractive error with males being more likely to have a refractive error than females $(p=0.02)$.

Conclusion: This study highlights the ongoing ophthalmic needs in a pediatric population in Sierra Leone. Examination of a larger cohort would better define the EVD-associated ocular complications in pediatric patients.

\section{P-30: Development of Text Messaging Intervention Content for Improving Eating Behaviors Among African American Men and Women}

Jylana L. Sheats, ${ }^{1}$ Donald Diego Rose, ${ }^{1}$ Lydia Bazzano, ${ }^{2}$ and Patrick Bordnick ${ }^{3}$

${ }^{1}$ Global Community Health \& Behavioral Sciences Department, Tulane University School of Public Health \& Tropical Medicine; ${ }^{2}$ Department of Epidemiology, Tulane University School of Public Health \& Tropical Medicine; and ${ }^{3}$ School of Social Work, Tulane University

Background: Text messaging interventions have demonstrated effectiveness for improving fruit and vegetable consumption. Relative to other populations, limited diet-related mHealth research has been conducted with African American (AA) men and women as intervention targets. Data show that AA have poorer diet quality compared to whites and Latinos. Given their increasing rates of mobile phone usage and inclination for accessing health information via mobile phones, AA may find mHealth support to be worthwhile.

Objective: To develop a diet-related 8-week text message program for AA adults in New Orleans, LA.

Methods: Theoretically-guided focus groups were conducted with the target population $(\mathrm{N}=31)$ around their diet-related perceptions and the acceptability and preferred features of mHealth support for healthy eating. Findings led to the development of 7 message types: 1) goal-setting, 2) self-regulation, 3) tips and recipes, 4) local resources, 5) testimonials 6) demonstrations, and 7) challenges - with a proportion of messages being faith-based. Messages were created using concepts and language used by and/or extracted from: focus group transcripts, an existing database, local organizations, the Bible, and commentary during faith-based events. Readability was assessed using online software. Messages were pilot-tested with members of the target population $(\mathrm{N}=5)$ to ensure relevance and understanding, and modified as necessary.

Results: Fifty-six one-way and two-way messages were created with Flesch-Kincaid readability scores $<5$ th grade.

Conclusions: A mHealth intervention was developed with iterative feedback from the target population. The intervention will be tested in a RCT with the aim of improving eating behaviors among AA men and women.

\section{P-31: Against the Clock: Morning Circadian Misalignment is Associated with Insulin Resistance in Adolescent Girls with Polycystic Ovarian Syndrome}

Stacey L. Simon (presenting author), ${ }^{1}$ Cecilia Diniz Behn, ${ }^{2}$ Yesenia Garcia-Reyes, ${ }^{1}$ Haseeb Rahat, ${ }^{1}$ Kenneth P. Wright, Jr., ${ }^{3,4}$ Kristen J. Nadeau, ${ }^{1}$ and Melanie Cree Green ${ }^{1}$

${ }^{1}$ Department of Pediatrics, University of Colorado Anschutz Medical Campus; ${ }^{2}$ Department of Applied Mathematics and Statistics, Colorado School of Mines; ${ }^{3}$ Department of Integrative Physiology, University of Colorado Boulder; and ${ }^{4}$ Division of Endocrinology, Metabolism, and Diabetes, University of Colorado Anschutz Medical Campus

Background: Polycystic Ovarian Syndrome (PCOS), characterized by hyperandrogenism and anovulation, is associated with increased risk of sleep disturbances. Adolescent girls report more sleep complaints and have higher rates of type 2 diabetes (T2D) compared to boys. Circadian misalignment is associated with insulin resistance (IR) in adults but has not been examined in girls with PCOS.

Objectives: To compare circadian timing and examine relationships with IR in girls with PCOS and obese controls.

Methods: Sixty-two girls with a diagnosis of PCOS and 33 controls with BMI $>90$ th \%ile for age/sex $(15.7 \pm 1.8$ years $)$ were recruited from a tertiary weight-management clinic. Following one week of home objective sleep monitoring with wrist actigraphy, participants had hourly-sampled evening and morning salivary melatonin and fasting AM hormone analysis in laboratory. Independent-samples t-tests compared differences in circadian timing between groups. Regression analyses between circadian and IR variables were examined.

Results: Girls with PCOS had later timing of melatonin offset, longer time between bedtime and melatonin offset, and longer duration of melatonin secretion compared to controls. Later melatonin offset time and longer duration of melatonin secretion were significantly associated with higher total testosterone. Greater morning circadian misalignment - high melatonin levels for longer time after waking - was associated with worse insulin sensitivity as assessed by HOMA-IR.

Conclusions: Adolescent girls with PCOS appear to be at greater risk for morning circadian misalignment and related poor health outcomes. Future research is needed investigating the impact of improved circadian timing on prevention and treatment of IR and T2D in girls with PCOS.

\section{P-32: Using Digital Health to Improve Adoption of the Dietary Approaches to Stop Hypertension (DASH) Diet}

Dori M. Steinberg (presenting author), ${ }^{1,2}$ Melissa C. Kay, ${ }^{2}$ Laura P. Svetkey, ${ }^{3}$ and Gary G. Bennett ${ }^{2,4}$ 
${ }^{1}$ School of Nursing, Duke University; ${ }^{2}$ Duke Global Digital Health Science Center, Duke University; ${ }^{3}$ Department of Medicine, Duke University; and ${ }^{4}$ Department of Psychology, Duke University

Background: Over 40 million women have high blood pressure (BP), yet adoption of the evidence-based DASH diet is poor. Innovative approaches that extend the reach of DASH interventions, such as digital health, are needed.

Objective: To determine the feasibility of a digital health intervention to improve DASH adherence among women with elevated BP.

Methods: In a 3-month randomized-controlled trial, we compared diet tracking using a publicly available smartphone diet-tracking app without (control) or with feedback on DASH adherence (intervention). Our intervention platform extracted cloud-based nutrient data from the app, compared it to DASH recommendations and sent automated personalized feedback text messages. The primary outcome is the number of days participants tracked diet. Exploratory outcomes are changes in DASH adherence score (range 0-9) and participant satisfaction.

Results: Women $(\mathrm{N}=59)$ were on average age $49.8 \pm 11.9$, primarily White $(72 \%)$, and college-educated $(83 \%)$. Average baseline DASH score was $2.2 \pm 1.6$. Over 3 months, intervention and control participants were similar in days tracked/week $(4.3 \pm 1.4$ vs. $4.6 \pm 1.4 ; \mathrm{p}=0.21)$ and changes in DASH score $(0.9 \pm 1.6$ vs. $1.0 \pm 1.0 ; \mathrm{p}=0.51)$. About half $(55 \%)$ thought the texts were very helpful; $79 \%$ would recommend this program to a friend.

Conclusions: Our cloud-based digital health intervention to improve DASH adherence among high risk women was feasible. Engagement was high with diet tracking; however, the intervention did not enhance DASH adherence over diet tracking alone in our small sample. Larger studies are needed to determine whether digital health interventions can improve population-level adoption of DASH and lead to demonstrable reductions in BP.

\section{P-33: Breastfeeding among Postpartum Incarcerated Women in the U.S.}

Carolyn Sufrin (presenting author), ${ }^{1,2}$ Lauren Beal, ${ }^{1}$ Jennifer G. Clarke, ${ }^{3}$ and William D. Mosher ${ }^{2}$

${ }^{1}$ Department of Gynecology \& Obstetrics, Johns Hopkins School of Medicine; ${ }^{2}$ Johns Hopkins Bloomberg School of Public Health; and ${ }^{3}$ Rhode Island Department of Corrections

Background: There are over 213,000 women behind bars in the U.S. Most are reproductive age; some enter jail or prison pregnant and may deliver in custody. Breastfeeding has wellestablished benefits and remains a consideration for postpartum incarcerated women. It is not known whether prisons and jails allow postpartum women to breastfeed, or how many women do.

Objective(s): To describe breastfeeding policies and frequency of breastfeeding at U.S. prisons and jails. Methods: We surveyed administrators at a geographically diverse sample of 22 state prison systems and 6 county jails about breastfeeding policies. These facilities also reported frequency of breastfeeding among postpartum women for 6 months.

Results: Seven prisons (32\%) and 5 jails (83\%) in our sample had written policies on breastfeeding. Eleven prisons $(50 \%)$ and one jail (17\%) did not allow incarcerated people to breastfeed or pump breast milk. Of the 330 known postpartum people in the 11 prisons that allowed breastfeeding during a 6 month period, only
$48(14 \%)$ were breastfeeding or pumping breast milk. In the 5 jails that allowed either breastfeeding or pumping breast milk, only a minority (25\%) of the 133 postpartum people were breastfeeding.

Conclusions: It is feasible for prisons and jails to enable postpartum women to provide breastmilk for their infants. Less than $1 / 3$ of prisons in our study had a written policy on breastfeeding. At sites that allowed breastfeeding, few postpartum women actually breastfed or pumped breastmilk. Interventions are needed to formalize policies and to promote breastfeeding for incarcerated postpartum women.

\section{P-34: Understanding Pain and Pain Treat Treatment Choices Among Older African American Women}

Janiece L. Taylor (presenting author), ${ }^{1,2}$ Laken Roberts, ${ }^{1}$ Brittany Drazich, ${ }^{1}$ Safiyyah M. Okoye, ${ }^{1}$ Jennifer Wenzel, ${ }^{1,2}$ and Sarah L. Szanton ${ }^{1,2}$

\section{${ }^{1}$ Johns Hopkins School of Nursing; and ${ }^{2}$ Johns Hopkins Center of Innovative Care in Aging}

Background: More than 50 million American adults are living with significant chronic or severe pain. When older African American women experience pain they have higher rates of undermanaged pain and subsequent physical function decline and pain related disability than other racial ethnic groups. It is important to understand the pain experience of older African American women in order to develop appropriate interventions to manage their pain.

Objective: The objective of this study was to identify how older African American women cope with their pain and barriers and facilitators to obtaining pain management.

Methods: A qualitative descriptive study was done with 20 community dwelling African American women who reported chronic pain. Their average age was 75.6 (SD 6.98). The women were recruited after completing or serving in the control group of a behavioral activation intervention, Community Aging in Place-Advancing Better Living for Elders ([CAPABLE] NIA: R01-AG040100, PI: Sarah Szanton). Qualitative content analysis was used to code the data line by line. Two of the coauthors coded the data to maintain validity. Codes were grouped and themes were inductively derived from the codes. Three of the coauthors agreed upon the themes.

Results: We identified four major themes in this study: "fear of treatment consequences," "resilience to pain," "depressive symptoms associated with pain," and "pain interferences with life."

Conclusions: The data from this study will be used to map a proposed intervention to target pain and depressive symptoms among community dwelling older African American women living with chronic pain and depressive symptoms.

\section{P-35: Adherence to the 2012 National Cervical Cancer Screening Guidelines in Women 30-65 Years of Age: A Retrospective Review}

Deanna Teoh, ${ }^{1}$ Gretchen Hultman, ${ }^{2}$ Melissa Geller, ${ }^{1}$ Eileen Harwood, ${ }^{3}$ Chap Le, ${ }^{4}$ Rachel Isaksson Vogel, ${ }^{1}$ Genevieve Melton-Meaux, ${ }^{2}$ and Shalini Kulasingam ${ }^{3}$

${ }^{1}$ Department of Obstetrics \& Gynecology, University of Minnesota; ${ }^{2}$ Department of Surgery, University of Minnesota; ${ }^{3}$ Division of Epidemiology \& Community Health, University of Minnesota; and ${ }^{4}$ Department of Biostatistics, University of Minnesota 
Objective: To determine the prevalence of cervical cancer over-screening (Pap test interval of $<3$ years) in women 30-65 years of age within Fairview Health System.

Methods: The electronic health record was queried to identify all women who had a screening Pap test between January 1, 2014-December 31, 2015 with an interval of $<3$ years since their previous test. A manual chart review was performed on a random $30 \%$ of charts to determine whether the Pap tests were indicated per the 2012 ASCCP management guidelines.

Results: A total of 3,300 Pap tests were reviewed. Of these, $2,311(70 \%$; 95\% CI $68.4-71.6 \%)$ were not indicated per the 2012 guidelines. The most common reasons for nonindicated tests included routine health maintenance $(56 \%)$, follow-up of previous abnormal result or cervical dysplasia surveillance which was inconsistent with the management guidelines (35\%); only $4 \%$ were done per patient request. On multivariate analysis of patient and provider characteristics, nurse practitioners (OR 1.34, 95\% CI 1.03-1.73) and Gynecologists (OR 1.71, 95\% CI 1.33-2.20) were more likely to over-screen. Over-screening was more likely in 2015 (OR 2.18, 95\% CI 1.86-2.54) and in clinics within 60 miles of the Twin Cities metro area (OR 2.12, 95\% CI 1.193.75).

Conclusions: Using conservative criteria, a majority of Pap tests in women between the ages of 30-65 years were not guideline adherent. Our data suggest that interventions at the provider level, especially among nurse practitioners and gynecologists, are needed to improve guideline-adherent cervical cancer screening in this age group.

\section{P-36: Exploring Testosterone Levels in Adolescent Boys Hospitalized for Medical Stabilization of an Eating Disorder}

C. Alix Timko (presenting author), ${ }^{1,2}$ Levi DeFilipp, ${ }^{1}$ Anushua Bhattacharya, ${ }^{1}$ and Rebecka Peebles ${ }^{2,3}$

${ }^{1}$ Department of Child and Adolescent Psychiatry and Behavioral Health, Children's Hospital of Philadelphia; ${ }^{2}$ Perlemean School of Medicine, University of Pennsylvania; and ${ }^{3}$ The Craig Dalsimer Division of Adolescent Medicine, Department of Pediatrics, Children's Hospital of Philadelphia

Background: Males are underrepresented in research on EDs, in particular the impact of EDs and malnutrition on the endocrine system. In order to fully explore sex differences in the impact of EDs on the hypothalamic-pituitary-gonadal axis in males with EDs, we must first have a broader understanding of impact of EDs on testosterone levels and its medical correlates.

Objectives: To characterize the relationship between testosterone levels and medical severity in males with EDs.

Methods: A retrospective chart review of males who were hospitalized for medical stabilization in the context of an ED at a pediatric hospital from January 1, 2011-June 30, 2017.

Results: Initial examination of the medical record indicated that 121 males were hospitalized for an ED during this period. The average BMI was 17.57 (SD +3.64) with a median of 16.91. The majority of the sample is nonHispanic $(87.9 \%)$ and white $(72.7 \%)$. Over half the sample $(52.9 \%)$ was diagnosed with anorexia nervosa. Data abstraction is on-going.
Conclusions: The sample is relatively large and should provide insight into the impact of malnutrion on testosterone. Data will provide a starting point for targeted hypotheses regarding the impact of low testosterone on disease severity, course, medical side effects (such as bone density), and neurodevelopment. The initial chart review focuses on males, however, should sufficient data be abstracted from the medical record, we can expand this study to examine sex differences in adolescents hospitalized for ED treatment.

\section{P-37: Innovative Intersection of Online Social Networking and Medical Research: The Mayo Clinic Spontaneous Coronary Artery Dissection Registry}

Marysia S. Tweet (presenting author), ${ }^{1}$ Susan Kok, ${ }^{2}$ and Sharonne N. Hayes, ${ }^{1}$

${ }^{1}$ Department of Cardiovascular Diseases, Mayo Clinic, Rochester, MN; and ${ }^{2}$ Department of Internal Medicine, Mayo Clinic, Rochester, MN

Background: Spontaneous coronary artery dissection (SCAD) is an increasingly recognized and important etiology of acute coronary syndrome and sudden death among young women.

Objective: Assess the use of peer to peer Internet social networking (ISN) to facilitate research and accelerate development of the Mayo Clinic "Virtual” Multicenter SCAD Registry and the Mayo Clinic's Genetic Investigations in Spontaneous Coronary Artery Dissection study.

Methods: Using ISNs as a platform to increase awareness of the current registries, we aimed to develop a robust database of persons with confirmed history of SCAD.

Results: Since study initiation in 2010, 933 SCAD participants have been recruited to the ongoing Mayo Clinic SCAD Registry and 764 participated in the genetics investigation arm in which 9 families have more than one person with SCAD. Most participants are women $(96.3 \%)$, White $(94.5 \%)$ with mean age of $49+9.6$ years when enrolled. Of those 332 respondents to dedicated ISN survey questions, many $(24.7 \%)$ learned of the study from their cardiologist in addition to ISNs such as Facebook (20.2\%) and WomenHeart's online community $(17.8 \%)$.

Conclusions: Awareness and peer-to-peer networking via ISNs can facilitate robust registry development over a short period of time despite limitations and challenges. While convenience, cost efficiency and access to participants are potentially positive aspects, concerns regarding patient protection, confidentiality, and ethical research practices are important to address. The potential for making positive impact using ISN is worthwhile of further exploration.

\section{P-38: Unique Dialysis Nonadherence Patterns in African Americans Could Predict Risk In End-Stage Renal Disease}

Ebele M. Umeukeje (presenting author), ${ }^{1}$ Deklerk Ngankam, ${ }^{2}$ Lauren Beach, ${ }^{3}$ Jennifer Morse, ${ }^{1}$ Thomas Stewart, ${ }^{1}$ and Kerri L. Cavanaugh ${ }^{1}$ ${ }^{1}$ Vanderbilt University Medical Center; ${ }^{2}$ Drexel University
College of Medicine; and ${ }^{3}$ Northwestern University 
Background and Objectives: Thrice weekly hemodialysis provides adequate urea clearance, however morbidity and mortality rates in End Stage Renal Disease (ESRD) remain unacceptably high due to nonadherence. African Americans (AAs) have a higher ESRD prevalence rate, higher dialysis nonadherence rates, and higher odds of hospitalization. We hypothesized that AAs will have unique patterns of dialysis nonadherence, which will differ by age, gender, and dialysis vintage (i.e. total duration receiving dialysis), and associate with hospitalizations and emergency room (ER) visits.

Methods: Three-month retrospective cohort of AAs receiving hemodialysis for $>90$ days. Dialysis attendance data was graphically displayed in a dot plot. T-test or Chi-square was used to examine associations of nonadherence with demographic factors, hospitalizations and ER visits (exploratory outcomes). Missed, shortened and completed treatments were grouped into six categorical patterns of nonadherence ranging from very good (zero missed/shortened treatment) to very poor (several consecutive missed/shortened treatments).

Results: There were 113 AAs (53\% female, mean age 57 years [SD 16], dialysis vintage 69 months [SD 65]). Shortened treatments associated with higher dialysis vintage (mean dialysis vintage in months [SD]: (87.9 [79.2] vs. 58.8 [53.4]; $\mathrm{p}=0.02$ ) while shortened treatments either alone or in combination with missed treatments associated with younger age (mean age in years [SD]: (55.6 [15.4] vs. 65.4 [16.2]; $\mathrm{p}=0.02)$. In exploratory analyses, we developed the hypothesis that the number of missed treatments is predictive of ER visits among men but not women.

Conclusions: Amongst AA dialysis patients, unique patterns of dialysis nonadherence could predict outcomes, and plausibly inform the development of targeted interventions to improve adherence in these vulnerable patients.

\section{P-39: Sex- and Non Sex-Specific Differences in Carbohydrate Tolerance During Remission in Patients with Ketosis-Prone Diabetes}

Priyathama Vellanki (presenting author), ${ }^{1}$ Darko Stefanovski, ${ }^{2}$ Isabel Anzola, ${ }^{1}$ Dawn D. Smiley, ${ }^{1}$ Limin Peng, ${ }^{3}$ and Guillermo E. Umpierrez ${ }^{1}$

${ }^{1}$ Division of Endocrinology, Metabolism and Lipids, Emory University School of Medicine, Atlanta GA; ${ }^{2}$ Department of Clinical Studies-New Bolton Center, University of Pennsylvania School of Veterinary Medicine, Kennett Square PA; and ${ }^{3}$ Rollins School of Public Health, Emory University, Atlanta GA

Background and Objectives: Ketosis-prone diabetes presents in obese African Americans and characterized by newonset DKA with 3:1 male:female prevalence. With intensive insulin treatment, many patients achieve near-normoglycemia remission (HbA1c $<7 \%$, fasting blood glucose [BG] $<130 \mathrm{mg}$ / dl). Glycemic status at remission varies from normal glucose tolerance (NGT), prediabetes or diabetes on oral glucose tolerance test (OGTT). We examined whether there were sex- and non sex-specific differences at remission with insulin sensitivity (Si) and secretion according to OGTT classification (American Diabetes Association) and hyperglycemia- free relapse (fasting $\mathrm{BG} \geq 130 \mathrm{mg} / \mathrm{dl}, \mathrm{HbA} 1 \mathrm{c}>7 \%$ or 2 random $\mathrm{BG}$ $\geq 180 \mathrm{mg} / \mathrm{dl}$ ).

Methods: Seventy-five subjects who presented with DKA and severe hyperglycemia (BG $>400 \mathrm{mg} / \mathrm{dl}$ without DKA) and attained remission $(n=45$ [men], $n=30$ [women]) underwent
2- hour 75-gm OGTT a week after remission with 336 days (median) follow-up. Si was calculated using OGTT minimal model analysis. Insulin secretion was calculated as incremental area under the curve of insulin (IncreAUCi) from OGTT. Disposition index (DI) was calculated as SixIncreAUCi.

Results: Baseline characteristics did not differ by sex. The distribution of OGTT classification was NGT (12\%), prediabetes $(45 \%)$ and diabetes $(\mathrm{n}=43 \%)$ with no differences by sex. DI was higher in patients with NGT vs prediabetes vs diabetes $(\mathrm{p}<0.001)$. Higher DI in NGT was explained by Si than IncreAUCi in NGT vs prediabetes vs diabetes ( $\mathrm{Si}: \mathrm{p}<0.001$; IncreAUCi: $p=0.14)$. Di, Si and IncreAUCi did not differ by sex. Multivariate analysis did not show significant association with sex and hyperglycemia relapse-free survival.

Conclusions: Attaining NGT at remission is characterized by higher insulin sensitivity rather than higher insulin secretion without sex-specific differences. Sex was not associated with hyperglycemia-free survival.

\section{P-40: Sex-Dependent Regulation of Autophagy by Midkine in Pediatric Dilated Cardiomyopathy}

Kathleen C. Woulfe (presenting author), ${ }^{1}$ Cortney E. Wilson, ${ }^{1}$ Xuan Jiang, ${ }^{1}$ Mark Y. Jeong ${ }^{1,2}$ Shelley D. Miyamoto, ${ }^{3}$ Brian L. Stauffer, ${ }^{1,2}$ and Carmen C. Sucharov ${ }^{1}$

${ }^{1}$ Department of Medicine, Division of Cardiology, University of Colorado Denver School of Medicine, Aurora, CO; ${ }^{2}$ Department of Medicine, Division of Cardiology, Denver Health and Hospital Authority, Denver, CO; and ${ }^{3}$ Department of Pediatrics, Division of Cardiology, University of Colorado Denver School of Medicine and Children's Hospital Colorado, Aurora, $\mathrm{CO}$

Background: Sex differences are observed in pre-pubertal pediatric patients with dilated cardiomyopathy (DCM), in particular, girls with DCM have worse outcomes than boys. One potential factor which may regulate sex differences in pediatric cardiac function is midkine (MDK), a protein that is highly elevated in the serum of pediatric DCM patients. While MDK's role in cardiac dysfunction is unclear, in cancer models, MDK has been found to inhibit autophagy.

Objective: The objective of this study was to determine if high exogenous MDK leads to sex differences in autophagy and cardiac function.

Methods: Young male and female mice were treated for 7 days with $3 \mathrm{mg} / \mathrm{kg} /$ week recombinant human MDK. Cardiac pathology was assessed by analysis of gene expression and protein changes, echocardiogram, and sarcomeric contractility and relaxation measured through myofibril mechanics.

Results: Female mice treated with MDK demonstrated upregulation of genes involved in the fetal gene program. Further, there were sex-specific differences in whole heart and subcellular function in MDK treated mice. Fractional shortening and rate of activation of contraction were decreased in female mice treated with MDK. In addition, myofibril relaxation mechanics were altered in female mice treated with MDK. Finally, analysis of mediators of autophagy indicate that MDK in female mice leads to inhibition of autophagy in the heart.

Conclusions: Elevated exogenous MDK in mice leads to sexspecific differences in cardiac function, sarcomeric mechanics, and alterations in autophagy. These findings suggest that MDK may contribute to worsened outcomes in pediatric girls with DCM. 


\section{P-41: Central Obesity Increases the Risk of Gestational Diabetes Partially Through Increasing Insulin Resistance}

Yeyi Zhu (presenting author), Monique M. Hedderson, Charles P. Quesenberry, Juanran Feng, and Assiamira Ferrara

Division of Research, Kaiser Permanente Northern California, Oakland, $C A$

Background: The role of central obesity, independent of overweight/obesity and other established risk factors, in gestational diabetes (GDM) risk remains understudied.

Objectives: We examined central obesity measures, waist-tohip ratio (WHR) and waist circumference (WC), in early pregnancy in relation to GDM risk and evaluated the potential for insulin resistance markers to mediate the central obesity-GDM association.

Methods: The prospective Pregnancy Environment and Lifestyle Study of 1,750 women recorded waist and hip circumferences at gestational weeks 10-13. In a nested casecontrol study within the cohort, 115 GDM cases and 230 controls had fasting insulin, homeostasis model assessmentof-insulin resistance (HOMA-IR), and adiponectin measurements at weeks 16-19. Poisson and conditional logistic regression models were used, adjusting for other risk factors (age $\geq 35$ years, minority race/ethnicity, prepregnancy overweight/obesity, family history of diabetes, previous GDM, and pre-existing hypertension).

Results: For women with WHR $<0.85$, one or more other risk factors increased GDM risk 1.99-fold (95\% CI 0.99-4.02). For women with WHR $\geq 0.85$, but no other risk factors, GDM risks increased 2.41-fold (1.14-5.06), and with other risk factors, 6.22-fold (3.49-11.10). Further, WHR significantly improved GDM risk prediction beyond other risk factors $(\mathrm{P}<0.001)$. Similar but attenuated results were observed for WC $\geq 88 \mathrm{~cm}$. Insulin, HOMA-IR, and adiponectin levels mediated the WHR-GDM association by $9.0 \%, 9.6 \%$, and $11.1 \%$, respectively; corresponding mediation proportions for the WC-GDM association were $40.0 \%, 41.1 \%$, and $35.4 \%$ (all Pvalues $<0.04)$.

Conclusions: Central obesity in early pregnancy represented a high-risk phenotype for GDM independent of other risk factors and may inform early screening and prevention strategies. 